\title{
Lagrangian circle actions
}

\author{
Clément Hyvrier
}

\begin{abstract}
We consider paths of Hamiltonian diffeomorphisms preserving a given compact monotone lagrangian in a symplectic manifold that extend to an $S^{1}$-Hamiltonian action. We compute the leading term of the associated lagrangian Seidel elements. We show that such paths minimize the lagrangian Hofer length. Finally, we apply these computations to lagrangian uniruledness and to give a nice presentation of the quantum cohomology of real lagrangians in monotone symplectic toric manifolds.
\end{abstract}

53D12, 53D20, 57R17, 57R58

\section{Introduction}

Let $\left(M^{2 n}, \omega\right)$ denote a symplectic manifold and let $L$ be a compact connected lagrangian in $M$. Here we will consider exact lagrangian loops of $L$. Consider the set of Hamiltonian isotopies starting at the identity and with ending point a Hamiltonian diffeomorphism preserving $L$ :

$$
\mathcal{P}_{L} \operatorname{Ham}(M, \omega):=\left\{\gamma:[0,1] \stackrel{C^{\infty}}{\longrightarrow} \operatorname{Ham}(M, \omega) \mid \gamma_{0}=\mathrm{id}, \gamma_{1}(L)=L\right\} .
$$

These are the paths generating exact lagrangian loops of $L$ (see M Akveld and D Salamon [1]). Similarly to loops of Hamiltonian diffeomorphisms, such paths define automorphisms of the lagrangian quantum homology of $L$ (see S Hu, F Lalonde, and $\mathrm{R}$ Leclercq [12]). Any such automorphism can be seen as multiplication by an invertible element of the lagrangian quantum homology, called the lagrangian Seidel element.

For weakly exact lagrangians it has been shown in [12] that the lagrangian Seidel morphism is always trivial, hence the Seidel element is simply given by the fundamental class of $L$.

In this paper we are interested in computing lagrangian Seidel elements for those paths admitting extensions to a loop of Hamiltonian diffeomorphisms coming from an $S^{1}$-action on $(M, \omega)$. In other words, for the elements in $\mathcal{P}_{L} \operatorname{Ham}(M, \omega)$ that are homotopic to paths which, when squared, yield an $S^{1}$-Hamiltonian action on the symplectic manifold. 
To ensure that all the automorphisms we want to compute are well-defined we will assume that $(M, L)$ is monotone. If $\mu_{L}: \pi_{2}(M, L) \rightarrow \mathbb{Z}$ denotes the Maslov index and if $I_{\omega}: \pi_{2}(M, L) \rightarrow \mathbb{R}$ is the $\omega$-valuation, then monotonicity means that $I_{\omega}=\lambda \mu_{L}$ for some real $\lambda>0$. We further ask that the minimal Maslov number of $L$, denoted $N_{L}$, is at least 2 , ie that

$$
N_{L}:=\inf _{\pi_{2}(M, L)}\left\{\mu_{L}(A) \mid \mu_{L}(A)>0\right\} \geq 2 .
$$

In this framework we will compute the leading term of the Seidel element. We will also show that in some cases all the other terms vanish. For instance, this is the case for the (monotone) totally real lagrangians in toric manifolds. This computation can be seen as the relative counterpart of the computation done by D McDuff and S Tolman [16] for the Seidel elements of an $S^{1}$-Hamiltonian action on $M$.

These calculations imply that such Hamiltonian paths cannot define null-homotopic exact lagrangian loops. We will further show that for paths giving $S^{1}$-Hamiltonian actions when squared, the lagrangian Hofer length is minimized, hence they define relative geodesics in their homotopy class with fixed endpoints. This is not that surprising considering that the obtained Hamiltonian loops define geodesics in their homotopy class, as shown by D McDuff and J Slimowitz [15]. We point out that such results can be useful for studying lagrangian unirulings, defined by P Biran and O Cornea [5]. The main class of examples for which we concretely apply the calculations mentioned above are the real lagrangians in monotone symplectic toric manifolds, that is, symplectic manifolds $\left(M^{2 n}, \omega\right)$ with a Hamiltonian action of $\mathbb{T}^{n}$ with some positivity assumption. These lagrangians are the fixed point sets of the unique antisymplectic involution preserving the moment map of the torus action. Under a monotonicity assumption, L Haug [10] showed that these lagrangian submanifolds are wide with respect to a $\mathbb{Z}_{2}$-Laurent polynomial coefficient ring. This means that the corresponding lagrangian quantum homology must split as a product of the $\mathbb{Z}_{2-}$ Morse homology of $L$ with the coefficient ring. We will show that the multiplicative quantum relations of $L$ are generated by lagrangian Seidel elements. This can be seen as a relative version of the observation made by McDuff and Tolman in [16]. Using Haug's result we then describe the lagrangian quantum homology as a quotient of some polynomial ring, analogously to the absolute case.

Formulation of the main result We need to introduce lagrangian quantum homology. Roughly speaking, this is the homology theory obtained by deforming the Morse differential on $L$ taking into account pseudoholomorphic disks in $M$ with boundary in $L$. More precisely, this is the homology of the pearl complex

$$
C\left(L ; f, g_{L} ; J ; \Lambda_{L}\right):=\left(R\langle\text { Crit } f\rangle \otimes \Lambda_{L}, d_{Q}\right),
$$


where $\left(f, g_{L}\right)$ is a Morse-Smale pair for $L, \Lambda_{L}:=R\left[q^{-1}, q\right]$ is the ring of $R$-Laurent polynomials graded by requiring that $|q|=1$ and where the differential $d_{Q}$ can be written as a sum of $\Lambda_{L}$-linear maps

$$
d_{Q}=d_{0}+d_{1} \otimes q^{-N_{L}}+d_{2} \otimes q^{-2 N_{L}}+\cdots,
$$

where $d_{0}$ stands for the Morse differential of $f$ and

$$
d_{k}: R\left\langle\mathrm{Crit}_{r} f\right\rangle \rightarrow R\left\langle\mathrm{Crit}_{r+k N_{L}-1} f\right\rangle
$$

is obtained by counting pearl trajectories, ie chains of gradient flow lines of $f$ and $J$-holomorphic disks in $M$ with boundary on $L$ with cumulative Maslov index $k N_{L}$. In the present text we will only consider $R=\mathbb{Z}_{2}$ as ground coefficients. From Biran and Cornea [4] the homology of this complex is generically well defined under the monotonicity assumption. We will denote by $\mathrm{QH}_{*}\left(L ; \Lambda_{L}\right)$ the corresponding lagrangian quantum homology. For more on this, we refer to [4] and the references therein.

lagrangian Seidel elements are invertible elements $S_{L}(\gamma) \in \mathrm{QH}_{*}\left(L ; \Lambda_{L}\right)$, where $\gamma \in \mathcal{P}_{L} \operatorname{Ham}(M, \omega)$. Their definition involves counting pearl trajectories with pseudoholomorphic sections in the Hamiltonian fibration $M \hookrightarrow P_{\gamma}^{\text {half }} \stackrel{\pi}{\longrightarrow} D^{2}$ associated with $\gamma$, whose boundary lies on the lagrangian $\pi^{-1}\left(\partial D^{2}\right)$ (see Section 3). In fact, the lagrangian Seidel elements are defined as the images under the lagrangian Seidel isomorphism of the fundamental class of $L$. The Seidel isomorphism is defined either on a covering of the set of homotopy classes with fixed endpoints in $\gamma \in \mathcal{P}_{L} \operatorname{Ham}(M, \omega)$ as in $\mathrm{Hu}$ and Lalonde [11], or by fixing a choice of section as in Lalonde, McDuff and Polterovich [13]. We adopt the latter point of view and discuss, to some extent, what happens when $\gamma$ generates an exact lagrangian loop of $L$ admitting an $S^{1}$-Hamiltonian action extension:

Definition 1.1 We say that $\gamma \in \mathcal{P}_{L} \operatorname{Ham}(M, \omega)$ extends to an $S^{1}$-Hamiltonian action if it is homotopic, relative to endpoints, to a path $\gamma^{\prime}:[0,1] \rightarrow \operatorname{Ham}(M, \omega)$ such that the concatenation of $\gamma^{\prime}$ with itself, $\left(\gamma^{\prime}\right)^{2}$, defines an $S^{1}$-Hamiltonian action. We denote by $\mathcal{P}$ the set of such paths.

In particular, if $H_{t}: M \rightarrow \mathbb{R}$ denotes the family of (normalized) Hamiltonian functions generating $\gamma^{\prime}$, then $\left(\gamma^{\prime}\right)^{2}$ is generated by a smooth time-independent (normalized) function $K: M \rightarrow \mathbb{R}$. Thus the (normalized) Hamiltonian generating $\gamma^{\prime}$ is also timeindependent and one has $K=2 H$. In the remainder of the article, unless otherwise mentioned, we will assume that $\gamma$ is $\gamma^{\prime}$, ie that $\gamma$ squares to an $S^{1}$-action. Let $F_{\max }$ denote the maximal fixed point set component of the $S^{1}$-action associated to $\left(\gamma^{\prime}\right)^{2}$. Throughout the paper we will restrict our attention to the following case: 
(A1) $F_{\max }$ is semifree, ie the action is semifree in a neighborhood of $F_{\max }$.

(A2) L intersects $F_{\max }$ cleanly, and the intersection $F_{\max }^{L}:=L \cap F_{\max }$ is a lagrangian submanifold of $F_{\max }$.

Remark 1.2 (1) Note that (A2) implies $\operatorname{dim} F_{\max }^{L}=\frac{1}{2} \operatorname{dim} F_{\max }$.

(2) If the gradient flow of $K$ is contained in $L$, then (A2) holds. Indeed, let $J$ be an $S^{1}$-invariant almost complex structure on $M$ compatible with $\omega$. At any $x \in F_{\max }$ we have the splitting

$$
T_{x} M=T_{x} F_{\max } \oplus N_{x} \cong \operatorname{ker}(1-d \gamma(x)) \oplus N_{x},
$$

where $N_{x}$ is the symplectic complement of $T_{x} F_{\max }=\operatorname{ker}(1-d \gamma(x))$. Since $J$ is $S^{1}$-invariant, $J$ is also split and so is the induced Hermitian metric $g_{J}:=\omega(\cdot, J \cdot)$. By the assumption, we have that $T_{x} L$ is compatible with that splitting, meaning that

$$
T_{x} L=T_{x} F_{\max }^{L} \oplus_{J}\left\{v \in T_{x} L \mid v \in N_{x}\right\} .
$$

Since $L$ is lagrangian, the terms in this direct sum are maximally isotropic subspaces of $T_{x} F_{\max }$ and $N_{x}$, respectively, and so the claim follows.

For a fixed point $x$, let $w(x)$ be the sum of the weights at $x$. We recall that for an $S^{1}$-invariant $\omega$-compatible almost complex structure on $M$, the action of $S^{1}$ on $T_{x} M \cong \mathbb{C}^{n}$ is conjugate to a product of circle actions $z \mapsto e^{2 \pi k_{j} t} z, t \in S^{1}$. Then the sum of the weights $w(x)=\sum k_{j}$ defines a locally constant function and as such only depends on the connected component of the fixed point set in which $x$ lies. We will denote by $w_{\max }$ the sum of the weights for points in the fixed point set $F_{\max }$. The main result of this paper is the following:

Theorem 1.3 Let $L$ be a monotone compact lagrangian submanifold of $(M, \omega)$. Let $\gamma \in \mathcal{P}$ satisfy the assumptions (A1) and (A2). The corresponding lagrangian Seidel element is given by

$$
S_{L}(\gamma)=\left[F_{\max }^{L}\right] \otimes q^{-w_{\max }}+\sum_{\left\{B \in \pi_{2}(M, L) \mid \mu_{L}(B)>0\right\}} a_{B} \otimes q^{-w_{\max }-\mu_{L}(B)},
$$

where $\left[F_{\max }^{L}\right]$ and $a_{B}$ are elements of $H_{*}\left(L ; \mathbb{Z}_{2}\right)$ with $\operatorname{deg}\left(a_{B}\right)=\operatorname{dim} F_{\max }^{L}+\mu_{L}(B)$. In particular, if codim $F_{\max }=2$ then all lower-order terms $a_{B}$ vanish.

As an example let us mention the case of half of a Hamiltonian loop fixing a given divisor $D$ (which corresponds to a facet of the moment polytope) in a monotone 
symplectic toric manifold, a special case of Fano ${ }^{1}$ symplectic toric manifolds. The endpoints of such path fix the real lagrangian in this manifold (eg consider a meridian $S^{1}$ in $S^{2}$ and the action of rotating around the poles) and assumptions (A1) and (A2) are verified. Hence, if the real lagrangian is monotone, one concludes that the corresponding lagrangian Seidel element is given by $[D \cap L] \otimes q^{-w_{\max }}$. It is worth noticing that lower-order terms may appear in situations that are reminiscent of those discussed in [16, Theorem 1.10, Example 5.6]. Such behavior is shown to occur in Example 5.9. One could wonder if the more general results in [16] still hold in the lagrangian context. Similarly, one could ask if the closed formulas obtained by S Anjos and R Leclercq in the NEF case [2] have lagrangian counterparts. Unfortunately, the techniques used here are not adapted to answer such questions as regularity of symmetric almost complex structures generally fails.

Theorem 1.3 can be applied to deduce some results about uniruledness of lagrangian submanifolds. As defined by Cornea and Biran in [5], a monotone lagrangian manifold $L$ in $M$ is said to be 1-uniruled, or uniruled, if there exists a Baire second category subset of families of almost complex structures of $M$ with the property that for each such almost complex structure there is a nonconstant pseudoholomorphic disk in $M$ with boundary on $L$ passing through any generic point of $L$.

Theorem 1.4 Let $L \subset M$ be a closed monotone lagrangian and suppose there is $\gamma \in \mathcal{P}$ such that the corresponding $S^{1}$-Hamiltonian action verifies hypotheses (A1) and (A2) and that $\operatorname{codim}_{M} F_{\max }=2$. Then $L$ is uniruled.

For example, any real monotone lagrangian with $N_{L} \geq 2$ in a toric manifold is lagrangian uniruled. Indeed, the fundamental class $[L]$ of such a lagrangian does not appear in any lagrangian Seidel element associated to an $S^{1}$-circle action fixing one of the codimension-one faces of the Delzant polytope of the toric manifold. Furthermore, lagrangian Seidel elements are all invertible. The claim then follows from Lemma 5.10.

Theorem 1.3 also implies that exact lagrangian loops respecting the assumptions of the Theorem cannot be null-homotopic since the higher order term is not $[L]$. We further show the following:

Theorem 1.5 Let $L \subset M$ be a closed monotone lagrangian. Suppose there exists $\gamma \in \mathcal{P}_{L} \operatorname{Ham}(M, \omega)$ such that $\gamma^{2}$ defines a semifree $S^{1}$-Hamiltonian action on $M$. Then the lagrangian path induced by $\gamma$ minimizes the Hofer length in its homotopy class with fixed endpoints.

\footnotetext{
${ }^{1}$ Fano means that the first Chern class of the tangent evaluates positively on any nontrivial class, or equivalently that the anticanonical bundle is ample. Note that a Fano Kähler manifold is deformation equivalent to a monotone one.
} 
The paper is organized as follows. In Section 2 we recall the definition of Hamiltonian fibrations associated to loops or paths of Hamiltonian diffeomorphisms. In Section 3 we introduce lagrangian Seidel elements. Section 4 is devoted to the proofs of Theorems 1.3 and 1.5. In Section 5 we apply our results to lagrangian uniruledness (we give the proof of Theorem 1.4) and we show that the multiplicative relations for the lagrangian quantum homology of real lagrangians in monotone symplectic toric manifolds are generated by lagrangian Seidel elements (more precisely, we prove Proposition 5.5).

Acknowledgements I would like to thank François Charette, Octav Cornea, Tobias Ekholm and Yasha Savelyev for useful discussions. I would also like to thank François Charette for his suggestions and comments on an earlier version of this note, that helped improve the presentation of the paper; in particular, for explaining to me how to simplify the assumptions in Theorem 1.4. Finally, the final version of the paper benefited from numerous useful comments and suggestions from the anonymous referee.

\section{Hamiltonian fibrations}

A Hamiltonian fibration $\pi: P \rightarrow B$ with fiber $\left(M^{2 n}, \omega\right)$, and compact symplectic base $\left(B, \omega_{B}\right)$, is a symplectic fibration whose structure group reduces to $\operatorname{Ham}(M, \omega)$. Such fibrations are naturally equipped with a family $\left\{\omega_{b}\right\}_{b \in B}$ of symplectic forms in the fibers $\pi^{-1}(b)$ induced by $\omega$. It was shown by V Guillemin, E Lerman, and S Sternberg [8], and by McDuff and Salamon in full generality, that Hamiltonian fibrations are symplectically trivial over the 1-skeleton of $B$ and that they admit an Erhesman connection on $T P$ whose holonomy around any loop is Hamiltonian. This latter condition can be formally expressed as follows: there exists a closed 2form $\tau \in \Omega^{2}(P)$ extending $\omega$. The corresponding horizontal distribution, ie a direct complement in $T P$ to the vertical subbundle Vert $:=\operatorname{ker} d \pi$, is given by

$$
\operatorname{Hor}_{\tau}(p):=\left\{w \in T_{p} P \mid \tau(w, v)=0 \text { for all } v \in \operatorname{Vert}_{p}=\operatorname{ker} d \pi(p)\right\} .
$$

Different connection forms $\tau$ as above may determine the same horizontal distribution. However, a unique choice can be made by requiring that $\pi_{*} \tau^{n+1}=0$, where $\pi_{*}$ denotes integration over the fibers. When this latter normalization condition is satisfied we say that $\tau$ is a coupling form. Note that such Hamiltonian fibrations admit symplectic structures

$$
\Omega_{c}:=\tau+c \pi^{*} \omega_{B}
$$

where $c$ is a sufficiently large strictly positive real number. 
Recall that an $\omega$-tame almost complex structure $J$ on a symplectic manifold $(M, \omega)$ is a smooth endomorphism of $T M$ such that

$$
(J(p))^{2}=-\mathrm{id}_{T_{p} M} \text { for all } p \in M \text { and } \omega(\cdot, J \cdot)>0 .
$$

Let $\mathcal{J}(M, \omega)$ denote the set of $\omega$-tame almost complex structures, which is contractible and nonempty [14, Chapter 2]. The symplectic manifold $\left(P, \Omega_{c}\right)$ admits $\Omega_{c}$-tame almost complex structures $J_{P}$ that are compatible with $\pi$ and $\tau$, or fibered, in the following sense:

- $d \pi \circ J_{P}=J_{B} \circ d \pi$, where $J_{B} \in \mathcal{J}\left(B, \omega_{B}\right)$,

- $J_{b}:=\left.J_{P}\right|_{\pi^{-1}(b)} \in \mathcal{J}\left(\pi^{-1}(b), \omega_{b}\right)$ for all $b \in B$,

- $J_{P}$ preserves the horizontal distribution induced by $\tau$.

Let $\mathcal{J}\left(P, \Omega_{c}, \tau, \pi\right)$ denote the set of such almost complex structures. In fact, for fixed $\tau$, any family $J=\left\{J_{b}\right\}_{b \in B}$ of $\omega_{b}$-tame almost complex structures and any given $J_{B}$ give rise to a unique fibered $J_{P} \in \mathcal{J}\left(P, \Omega_{c}, \tau, \pi\right)$ for some $c \in \mathbb{R}$.

Hamiltonian fibrations as above are given two canonical cohomology classes. The first one is the vertical first Chern class induced by any family of almost complex structures $\left\{J_{b}\right\}_{b \in B}$ and defined by

$$
c_{v}:=c_{1}(\text { Vert }) \in H^{2}(P, \mathbb{Z}) .
$$

The second one is the de Rham cohomology class of the coupling form $[\tau] \in H^{2}(P, \mathbb{R})$ : this is the unique class such that

$$
\iota^{*}[\tau]=[\omega] \text { and }[\tau]^{n+1}=0 .
$$

In what follows we shall only consider Hamiltonian fibrations over $D^{2}$ and $S^{2}$.

\section{Hamiltonian fibrations associated to a loop of Hamiltonian diffeomorphisms}

Let $\mathcal{L} \operatorname{Ham}(M, \omega)$ be the set of loops of Hamiltonian diffeomorphisms based at the identity. Any element $\gamma_{t} \in \mathcal{L} \operatorname{Ham}(M, \omega)$ defines a Hamiltonian fibration over $S^{2}$ via the clutching construction. Namely, let $D^{+}$and $D^{-}$denote the unit disks in $\mathbb{C}$, but with opposite orientations. Then set

$$
P_{\gamma}:=\left(D^{+} \times M\right) \sqcup\left(D^{-} \times M\right) /\left(e^{i 2 \pi t}, x\right) \sim\left(e^{i 2 \pi t}, \gamma_{t}(x)\right), \quad t \in[0,1] .
$$

This is obviously a Hamiltonian fibration over $\left(S^{2}, \omega_{\mathrm{FS}}\right)$, where $\omega_{\mathrm{FS}}$ denotes the Fubini-Study form on $S^{2}$ with total area 2. Its isomorphism class only depends on the 
homotopy class of the loop $\left\{\gamma_{t}\right\}$. In fact, any Hamiltonian fibration over $S^{2}$ can be obtained in this way [13]. If $\tau_{\gamma}$ denotes the corresponding coupling form, then

$$
\Omega_{c}:=\tau_{\gamma}+c \pi^{*} \omega_{\mathrm{FS}}
$$

is symplectic for a sufficiently large positive constant $c$.

When $\left\{\gamma_{t}\right\}$ is given by an $S^{1}$-action on $F, P_{\gamma}$ can be described in the following way. Let $p: S^{3} \rightarrow S^{2}$ denote the Hopf fibration. The product $S^{3} \times M$ admits the free $S^{1}$-action

$$
e^{i t} \cdot\left(\left(z_{0}, z_{1}\right), x\right)=\left(\left(e^{-i t} z_{0}, e^{-i t} z_{1}\right), \gamma_{t}(x)\right),
$$

and the quotient $S^{3} \times{ }_{S^{1}} M$ can be identified with $P_{\gamma}$. In that setting, the coupling form is obtained by considering a connection 1-form on $S^{3}$. Namely, let $\alpha \in \Omega^{1}\left(S^{3}\right)$ be the standard contact structure, so that $d \alpha=p^{*} \omega_{\mathrm{FS}}$, where $\omega_{\mathrm{FS}}$ is the Fubini-Study form on $S^{2}$ normalized to have area 1. Let also $K: S^{3} \times M \rightarrow \mathbb{R}$ denote the moment map of the circle action above. Then $\omega-d(K \alpha) \in \Omega^{2}\left(S^{3} \times M\right)$ defines a closed basic form, hence defines a closed 2 -form on the quotient:

$$
\tau_{\gamma}=\operatorname{pr}_{*}(\omega-d(K \alpha)) \in \Omega^{2}\left(S^{3} \times_{S^{1}} M\right),
$$

where pr: $S^{3} \times M \rightarrow S^{3} \times S^{1} F$ denotes the projection and $\mathrm{pr}_{*}$ denotes integration over the fibers of pr. This form clearly extends $\omega$ on the fiber $F$, and since $K$ is normalized the integral $\pi_{*} \tau_{\gamma}^{n+1}$ vanishes. Thus $\tau_{\gamma}$ is a coupling form.

In this particular framework, each fixed point of the circle action yields a section of $P_{\gamma}$. Namely, for $x \in \operatorname{Fix}(\gamma)$, the corresponding section is

$$
\sigma_{x}:=S^{3} \times S^{1}\{x\} .
$$

The following will be useful later on:

Lemma 2.1 [16, Lemma 2.2] If $x$ is a fixed point of the Hamiltonian circle action of $\gamma$, then

$$
c_{v}\left(\sigma_{x}\right)=w(x) \text { and } \quad \tau_{\gamma}\left(\sigma_{x}\right)=-K(x),
$$

where $w(x)$ denotes the sum of the weights at $x$. Moreover, if $B$ is the class of a sphere formed by the $\gamma$-orbit of an arc between $x$ and $y$, then $B=\sigma_{x}-\sigma_{y}$.

Consider now an $S^{1}$-invariant $\omega$-tame almost complex structure on $M$. Note that the standard complex structure $J_{0}$ in $\mathbb{C}^{2}$ is $S^{1}$-invariant. Its restriction to $S^{3}$ preserves the contact structure $\operatorname{ker} \alpha$. Let $R$ denote the Reeb vector field associated to $\alpha$, and let $X_{K}$ denote the Hamiltonian vector field of the $S^{1}$-action on $M$. Then any vector 
field $v=\left[v_{1}, v_{2}\right] \in T\left(S^{3} \times_{S^{1}} M\right)$ admits a unique representative in $T\left(S^{3} \times M\right)$ lying in $\operatorname{ker} \alpha \oplus T M$, given by

$$
\left(v_{1}-\alpha\left(v_{1}\right) R, v_{2}+\alpha\left(v_{1}\right) X_{K}\right) .
$$

It follows that $J_{0} \times J$ descends to a well-defined almost complex structure $\bar{J}$ on $P_{\underline{\gamma}}$, which is obviously fibered and tames $\Omega_{c}$ for $c>\max K$. Note that $\sigma_{x}$ is then $\bar{J}_{-}$ holomorphic.

\section{Hamiltonian fibrations associated to $\gamma \in \mathcal{P}_{L} \operatorname{Ham}(M, \omega)$}

To any path $\gamma \in \mathcal{P}_{L} \operatorname{Ham}(M, \omega)$ one associates a Hamiltonian fibration over the 2-disk as follows. Let $\mathbb{H}$ denote the upper half plane in $\mathbb{C}$, and set

$$
\begin{aligned}
& D_{+}^{+}:=\{z=x+i y \in \mathbb{H}|| z \mid \leq 1\}, \\
& D_{-}^{+}:=\{z=x+i y \in \mathbb{H}|| z \mid \geq 1\} .
\end{aligned}
$$

Thus, the upper-half plane $\mathbb{H}$ is identified with $D_{+}^{+} \cup_{\varphi} D_{-}^{+}$, where the two regions are glued along their common boundary via the map

$$
\varphi: D_{+}^{+} \rightarrow D_{-}^{+}, \quad z \mapsto \bar{z}^{-1} \text {. }
$$

Let $\overline{\mathbb{H}}$ be the compactification of $\mathbb{H}$ by $\mathbb{R} \cup\{\infty\}$. Then $\overline{\mathbb{H}}$ can be identified with $D^{2}$, the disk of radius 1 .

The Hamiltonian fibration associated to the path is then given by the half clutching construction:

$$
P_{\gamma}^{\text {half }}:=\left(D_{+}^{+} \times M\right) \sqcup\left(D_{-}^{+} \times M\right) /\left(e^{i \pi t}, x\right) \sim\left(e^{i \pi t}, \gamma_{t}(x)\right) .
$$

Again, its isomorphism class only depends on the homotopy class with fixed endpoints of $\gamma_{t}$. This manifold carries symplectic structures such that the subbundle defined by collecting all the copies of $L$ along the boundary of $\overline{\mathbb{H}}$ is a lagrangian submanifold $N$ fibering over $S^{1}$. If $\gamma=\left\{\gamma_{t}\right\} \in \mathcal{P} \operatorname{Ham}_{L}(M, \omega)$, then

$$
N \cong \bigcup_{t \in[0,1]}\left\{e^{2 \pi i t}\right\} \times \gamma_{t}(L)
$$

This lagrangian actually embeds in $P_{\gamma}^{\text {half }} \cong D^{2} \times M$ and is called an exact lagrangian loop (see [1;12]). Considering the specific case where $\gamma \in \mathcal{P}$, one easily sees that $N$ is a lagrangian.

A connection form on $P_{\gamma}^{\text {half }}$ is given by

$$
\tau_{\gamma}=\omega-d H \wedge d x-d H \wedge d y-\frac{H}{\pi} d x \wedge d y,
$$


and symplectic structures are explicitly given by

$$
\Omega_{c}=\tau_{\gamma}+\frac{c}{\pi} d x \wedge d y, \text { for } c>0 \text { big enough. }
$$

One verifies that the parallel transport of $\tau_{\gamma}$ preserves the fiber bundle $N$. Letting $L_{S}$ be the copy of $L$ lying in the fiber over $s \in \partial D^{2}$, this is the same as saying that the vector field

$$
\left(s_{0},\left.\frac{d}{d s}\right|_{s=0} \gamma_{s}(p)\right), \quad p \in L_{s_{0}}
$$

along $N$ is horizontal with respect to $\tau_{\gamma}$. Hence, $N$ is a lagrangian submanifold of $P_{\gamma}^{\text {half }}$ for the symplectic forms $\Omega_{c}$. Note also that $\tau$ vanishes on $N$. We will denote by $\mathcal{T}(\gamma)$ the set of connection 2 -forms on $P_{\gamma}^{\text {half }}$ whose parallel transport along the boundary preserves $N$. Equivalently, these are the connection 2-forms that vanish identically on $N$ (see [1, Lemma 3.1]). The set of relative cohomology classes $[\tau] \in H^{2}\left(D^{2} \times M, N ; \mathbb{R}\right)$ associated to elements $\tau \in \mathcal{T}(\gamma)$ is a 1-dimensional affine space: for any $\tau_{0}, \tau_{1} \in \mathcal{T}(\gamma)$,

$$
\left[\tau_{1}\right]-\left[\tau_{0}\right]=c\left(\tau_{1}, \tau_{0}\right)\left[\frac{1}{\pi} d x \wedge d y\right], \quad s\left(\tau_{1}, \tau_{0}\right) \in \mathbb{R} .
$$

Hence, for $c$ big enough, $\tau=\tau_{0}+(c / \pi) d x \wedge d y$ is symplectic, and for $c$ small enough, $-\tau$ is symplectic. Set $\mathcal{T}^{ \pm}(\gamma):=\left\{\tau \in \mathcal{T}(\gamma) \mid \pm \tau^{n+1}>0\right\}$. Then any value of $c$ for which $\tau$ is nonsymplectic lies between the two real numbers

$$
\begin{aligned}
& \epsilon^{+}\left(\tau_{0}, N\right):=\inf \left\{c\left(\tau, \tau_{0}\right) \mid \tau \in \mathcal{T}^{+}(\gamma)\right\}, \\
& \epsilon^{-}\left(\tau_{0}, N\right):=\inf \left\{c\left(\tau, \tau_{0}\right) \mid \tau \in \mathcal{T}^{-}(\gamma)\right\} .
\end{aligned}
$$

The width of the corresponding nonsymplectic interval $\epsilon(N)$ does not depend on the reference point $\tau_{0}$, and is given by

$$
\epsilon(N)=\epsilon^{+}\left(\tau_{0}, N\right)-\epsilon^{-}\left(\tau_{0}, N\right) .
$$

\section{Hofer length of exact lagrangian loops}

Let $\gamma=\left\{\gamma_{t}\right\} \in \mathcal{P}_{L} \operatorname{Ham}(M, \omega)$. As seen above, this defines an exact lagrangian loop $N \subset S^{1} \times M$. Assume $\gamma_{t}$ is generated by the family $H_{t}$ of Hamiltonians. In the case where $M$ is not compact these must be compactly supported. The Hofer length of $N$ is defined to be

$$
\ell(N)=\int_{0}^{1}\left(\max _{x \in L_{t}} H_{t}(x)-\min _{x \in L_{t}} H_{t}(x)\right) d t,
$$

where $L_{t}=\gamma_{t}(L)$. Subsequently we will consider minimizing the Hofer length within the isotopy class of $\gamma$ with fixed endpoints. This is the same as minimizing $\ell(N)$ 
within its isotopy class of exact lagrangian loops. In other words, we will examine the quantity

$$
v(N):=\inf _{N^{\prime}} \ell\left(N^{\prime}\right)
$$

where the infimum is taken over all exact lagrangian loops that are Hamiltonian-isotopic to $N$. The following theorem is due to Akveld and Salamon:

Theorem 2.2 [1, Theorem B] For every exact lagrangian loop $N$,

$$
\epsilon(N) \leq v(N)
$$

\section{The doubling procedure}

Let $\gamma^{2}$ denote the loop of Hamiltonian diffeomorphisms associated to the path $\gamma \in \mathcal{P}$. First note that we have an obvious embedding

$$
\iota_{2}: P_{\gamma}^{\text {half }} \hookrightarrow P_{\gamma^{2}} .
$$

Taking the pullback of $\operatorname{pr}_{*}(\omega-d(K \alpha))$ under $\iota_{2}$ actually yields $\tau_{\gamma}$ in $(2-1)$.

In fact, $P_{\gamma^{2}}$ is made of two copies of $P_{\gamma}^{\text {half }}$ glued together along their boundaries. More precisely,

$$
P_{\gamma^{2}}=P_{\gamma}^{\text {half }} \cup_{\varphi} P_{\gamma}^{\text {half }}
$$

where

$$
\varphi: \partial P_{\gamma}^{\text {half }} \rightarrow \partial P_{\gamma}^{\text {half }}, \quad(s, x) \mapsto\left(-s, \gamma_{1}(x)\right)
$$

This happens to be useful subsequently to induce information on $P_{\gamma}^{\text {half }}$ from $P_{\gamma^{2}}$. In particular, this will be handy when dealing with holomorphic sections. Concerning sections, let us note that not only any section in $P_{\gamma^{2}}$ gives rise to a section in $P_{\gamma}^{\text {half }}$ (which may not have boundary on the lagrangian), but also, any section

$$
\sigma:\left(D^{2}, S^{1}\right) \rightarrow\left(P_{\gamma}^{\text {half }}, N\right), \quad z \mapsto(z, u(z))
$$

can be doubled to give a section in $P_{\gamma^{2}}$ with the equator being constrained to the lagrangian $\iota_{2}(N)$. In fact, identify $D^{2}$ with $\overline{\mathbb{H}}$. Note that $S^{2}$ is obtained by gluing $\overline{\mathbb{H}}$ and $e^{i \pi} \cdot \overline{\mathbb{H}}$ along their boundaries using the identification $s \mapsto-s$. Then the new section is given by

$$
\sigma_{\mathrm{db}}: S^{2} \rightarrow P_{\gamma^{2}}, \quad z \mapsto \begin{cases}(z, u(z)) & \text { if } z \in \overline{\mathbb{H}}, \\ \left(z, \gamma_{1}\left(u\left(e^{-i \pi} z\right)\right)\right) & \text { if } z \in e^{i \pi} \cdot \overline{\mathbb{H}} .\end{cases}
$$

Note that this is well-defined and continuous. 


\section{Lagrangian Seidel elements}

Here we recall the definition of Seidel elements in the relative case.

\section{Section classes and vertical Maslov index}

Consider the pair $\left(P_{\gamma}^{\text {half }}, N\right)$, as above. We say that $\sigma \in \pi_{2}\left(P_{\gamma}^{\text {half }}, N\right)$ is a section class if and only if $\pi_{*}(\sigma)=\left[D^{2}, S^{1}\right]$ is the positive generator. We say that it is a fiber class if $\sigma$ lies in the image of the inclusion map

$$
\pi_{2}(M, L) \rightarrow \pi_{2}\left(P_{\gamma}^{\text {half }}, N\right),
$$

thus implying $\pi_{*} \sigma=0$. As shown in [12], the following sequence is exact in the middle:

$$
\pi_{2}(M, L) \rightarrow \pi_{2}\left(P_{\gamma}^{\text {half }}, N\right) \rightarrow \pi_{2}\left(D^{2}, S^{1}\right)
$$

Definition 3.1 [12] Let $u: D^{2} \rightarrow P_{\gamma}^{\text {half }}$ be a smooth map representing an element $\sigma \in \pi_{2}\left(P_{\gamma}^{\text {half }}, N\right)$. The vertical Maslov index of $B$ is the Maslov index of the pair $\left(u^{*}\left(T^{v} P_{\gamma}^{\text {half }}\right), u^{*} T^{v} N\right)$, where $T^{v} N$ denotes the vertical tangent bundle of the bundle $N$. We will denote this number by $\mu_{\gamma}^{v}(\sigma)$ or $\mu_{v}$ for simplicity.

The vertical Maslov index is a well-defined $\mathbb{Z}$-valued morphism on $\pi_{2}\left(P_{\gamma}^{\text {half }}, N\right)$, which further satisfies that

$$
\mu_{N}(\sigma)=\mu_{\gamma}^{v}(\sigma)+2 \quad \text { and } \quad \mu_{N}\left(\sigma-\sigma^{\prime}\right)=\mu_{L}\left(\sigma-\sigma^{\prime}\right)
$$

for two section classes $\sigma$ and $\sigma^{\prime}$. The next lemma follows directly from the definitions of the vertical Maslov class and of the doubling of a section:

Lemma 3.2 Let $\gamma^{2}$ denote the loop of Hamiltonian diffeomorphisms associated to the path $\gamma \in \mathcal{P}$. If $\sigma$ represents the class $[\sigma] \in \pi_{2}\left(P_{\gamma}^{\text {half }}, N\right)$ then $\sigma_{\mathrm{db}}$ represents the class $\left[\sigma^{2}\right]:=[\sigma \# \sigma] \in \pi_{2}\left(P_{\gamma^{2}}\right)$, and one has

$$
c_{v}\left(\left[\sigma_{\mathrm{db}}\right]\right)=\mu_{\gamma}^{v}([\sigma]) \quad \text { and } \quad \tau_{\gamma^{2}}\left(\left[\sigma_{\mathrm{db}}\right]\right)=2 \tau_{\gamma}([\sigma]) .
$$

Proof The second equality follows by definition. For the first equality,

$$
2 c_{v}\left(\left[\sigma^{2}\right]\right)=\mu_{v}\left(\left[\sigma^{2}\right]\right)=\mu_{v}([\sigma])+\mu_{v}([\sigma])=2 \mu_{v}([\sigma]),
$$

hence the claim. 


\section{Holomorphic and antiholomorphic sections}

Let $j$ denote the standard complex structure on the disk, that is, the anticlockwise rotation by 90 degrees in the plane. Let $\left\{J_{z}\right\}, z \in D^{2}$, denote a smooth family of $\omega$-tamed almost complex structures in $M$. Let

$$
\mathbb{H}:=H \otimes d x+H \otimes d y .
$$

This is a 1 -form over $D^{2}$ with values in $C_{0}^{\infty}(M)$. Let $X_{\mathbb{H}}$ be the induced 1 -form with values in Hamiltonian vector fields of $M$, and let $X_{\mathbb{H}}^{0,1}$ denote the corresponding $(j, J)$ antiholomorphic part. These data provide an almost complex structure on $P_{\gamma}^{\text {half }}$, as follows:

$$
J_{P}(\tau, J)(z, x):=\left(\begin{array}{cc}
j(z) & 0 \\
X_{\mathbb{H}}^{0,1}(z, x) & J_{z}(x)
\end{array}\right) .
$$

It is easy to check that this is fibered. Furthermore, if $\tau \in \mathcal{T}^{ \pm}(\gamma)$ then $J_{P}(\tau, \pm J)$ is $\pm \tau$-tamed. In fact $J_{P}(\tau, J)$ is $\Omega_{c}$-tamed for $c$ large enough.

We consider the following boundary value problem for smooth sections $u: D^{2} \rightarrow P_{\gamma}^{\text {half }}$ :

$$
J_{P} \circ d u=d u \circ j \quad \text { and } \quad u\left(\partial D^{2}\right) \subset N .
$$

Fix a section class $A$ and let $\mathcal{M}\left(P_{\gamma}^{\text {half }}, A ; \tau, J\right)$ denote the moduli space of $J_{P}(\tau, J)$ holomorphic sections representing $A$ :

$$
\mathcal{M}\left(P_{\gamma}^{\text {half }}, A ; \tau, J\right):=\left\{u: D^{2} \rightarrow P_{\gamma}^{\text {half }} \mid u \text { satisfies (3-2) and }[u]=A\right\} .
$$

For generic $(\tau, J)$ this is a manifold of dimension $n+\mu_{v}(A)[1 ; 11]$. Taking $-J$ instead of $J$, the moduli space $\mathcal{M}\left(P_{\gamma}^{\text {half }}, A ; \tau,-J\right)$ is similarly defined and is generically a manifold of dimension $n-\mu_{v}(A)$.

Remark 3.3 Note that a fixed point $x \in L$ of $\gamma$ defines a section of $P_{\gamma}^{\text {half }}$, given by

$$
u: D^{2} \rightarrow P_{\gamma}^{\text {half }}, \quad z \mapsto(z, x) .
$$

This section is $J_{P}(\tau, \pm J)$-holomorphic. Indeed, for $z=s+i t$ and for $u=(z, \tilde{u})$, the first part of (3-2) is equivalent to

$$
\frac{\partial \tilde{u}}{\partial s}+J_{z}(\widetilde{u}) \frac{\partial \tilde{u}}{\partial t}+X_{H}(\tilde{u})-J_{z}(\widetilde{u}) X_{H}(\widetilde{u})=0 .
$$

If $x \in L$ is fixed under $\gamma$, then $\tilde{u}(s, t)=x$, so that $\partial \tilde{u} / \partial s=\partial \tilde{u} / \partial t=0$. Furthermore, $X_{H}(\tilde{u})=0$ since $x$ is a fixed point. 


\section{The relative Seidel element}

We now define the relative Seidel element associated to a path $\gamma \in \mathcal{P}_{L} \operatorname{Ham}(M, \omega)$. First, we recall the definition of the lagrangian Seidel morphism given in [12]. Consider a Morse-Smale pair $(F, G)$, where $F \in C^{\infty}(N)$ is a Morse function and $G$ is a metric on $N$ such that:

(1) $f_{ \pm 1}:=\left.F\right|_{L \pm 1}$ are Morse functions on the fibers $L_{ \pm 1}$ over \pm 1 of $N$.

(2) Crit $f_{+} \cup$ Crit $f_{-}=$Crit $F$.

(3) $\max f_{-}+1<\max f_{+}$.

(4) There exist neighborhoods $U_{ \pm} \cong(-\epsilon, \epsilon)$ of $\pm 1 \in S^{1}$ trivializing the fiber bundle $N$, with respect to which $\left.F\right|_{U_{ \pm}}(t, x)=f_{ \pm}(x)+\varphi_{ \pm}(t)$ for any $(t, x) \in U_{ \pm} \times L_{ \pm 1}$ and where $\varphi_{ \pm}: U_{ \pm} \rightarrow \mathbb{R}$ are quadratic and $\varphi_{+}$is of index 1 at 0 while $\varphi_{-}$is of index 0 at 0 .

(5) We also ask that $\left.G\right|_{U_{ \pm}}$is a product metric $d t^{2}+G_{ \pm}$and that $\left(f_{ \pm}, G_{ \pm}\right)$are Morse-Smale pairs.

Such pairs $(F, G)$ can be chosen generically.

Fix $\tau \in \mathcal{T}(\gamma)$ and a family $J=\left\{J_{z}\right\}_{z \in D^{2}}$ of $\omega$-tamed almost complex structures on $M$. Let $J_{P}(\tau, J)$ be the corresponding fibered almost complex structure on $P_{\gamma}^{\text {half }}$. For $\sigma$ a section class and for $x_{-} \in$ Crit $f_{-}$and $x_{+} \in$ Crit $f_{+}$, let

$$
\mathcal{M}^{\text {pearl }}\left(x_{-}, x_{+}, \sigma ; \tau, J, F, G\right)
$$

denote the set of pearl trajectories from $x_{-}$to $x_{+}$representing $\sigma$. In particular, elements of this moduli space have one $J_{P}(\tau, J)$-holomorphic section component with boundary on $N$, and possibly many $J_{ \pm 1}$-holomorphic disk components with boundary on $L_{ \pm 1}$.

For simplicity we will omit the auxiliary data $\tau, J, F, G$ in the notation. This set is a manifold of dimension

$$
\operatorname{dim} \mathcal{M}^{\text {pearl }}\left(x_{-}, x_{+}, \sigma\right)=\left|x_{-}\right|_{N}-\left|x_{+}\right|_{N}+\mu_{N}(\sigma)-1=\left|x_{-}\right|_{L}-\left|x_{+}\right|_{L}+\mu_{v}(\sigma) .
$$

Fix a section class $\sigma_{0}$. As seen in the preceding section, any other section class can be written as $\sigma_{0}+B$ for some fiber class $B \in \pi_{2}(M, L)$. The lagrangian Seidel morphism is defined as follows:

Definition 3.4 [12] For $\gamma \in \mathcal{P}_{L} \operatorname{Ham}(M, \omega)$ and $\sigma_{0}$ a choice of a reference section class, the lagrangian Seidel morphism associated to $\gamma$ is an endomorphism of lagrangian 
quantum homology defined at the chain level by

$$
\begin{gathered}
S_{L}\left(\gamma, \sigma_{0}\right): R\left\langle\mathrm{Crit}_{\star} f_{-}\right\rangle \rightarrow R\left\langle\mathrm{Crit}_{\star} f_{+}\right\rangle, \\
S_{L}\left(\gamma, \sigma_{0}\right)\left(x_{-}\right):=\sum_{B, x_{+}} \#_{\mathbb{Z}_{2}} \mathcal{M}^{\text {pearl }}\left(x_{-}, x_{+}, \sigma_{0}+B\right) x_{+} q^{-\mu_{v}\left(\sigma_{0}\right)-\mu_{L}(B)},
\end{gathered}
$$

where the sum runs over all fiber classes $B \in \pi_{2}(M, L)$ and critical points $x_{+} \in \mathrm{Crit}_{\star} f_{+}$ such that $\left|x_{+}\right|_{L}=\left|x_{-}\right|_{L}+\mu_{v}\left(\sigma_{0}+B\right)$.

This only depends on the homotopy class of paths with fixed endpoints of $\gamma$. Moreover, since $L$ is monotone with $N_{L} \geq 2$, the lagrangian Seidel morphism is a chain morphism (with respect to the pearl differential) and is generically well-defined with respect to the data of $J_{P}, F$ and $G[5 ; 12]$. Since it is a chain morphism and since $\left[L_{-}\right]$, the maximum of Crit $f_{-}$, defines a pearl cycle, $S_{L}\left(\gamma, \sigma_{0}\right)\left(\left[L_{-}\right]\right)$is also a cycle.

Definition 3.5 For $\gamma \in \mathcal{P}_{L} \operatorname{Ham}(M, \omega)$ and $\sigma_{0}$ a choice of reference section class, the lagrangian Seidel element associated to $\gamma$ and $\sigma_{0}$ is the homology class in $\mathrm{QH}_{n}\left(L_{+} ; \Lambda_{L_{+}}\right)$given by

$$
\left[S_{L}\left(\gamma, \sigma_{0}\right)\left(\left[L_{-}\right]\right)\right]:=\left[\sum_{B, x_{+}} \#_{\mathbb{Z}_{2}} \mathcal{M}^{\text {pearl }}\left(\left[L_{-}\right], x_{+}, \sigma_{0}+B\right) x_{+} q^{-\mu_{v}\left(\sigma_{0}+B\right)}\right],
$$

where the sum runs over all fiber classes $B$ and critical points $x_{+} \in \mathrm{Crit}_{\star} f_{+}$such that $\left|x_{+}\right|_{L}=n+\mu_{v}\left(\sigma_{0}+B\right)$.

Below, and in fact in the statement of Theorem 1.3, we choose $\sigma_{0}$ to be $\sigma_{\max }$, the section class associated to a fixed point $x \in F_{\max }$. The reference to $\sigma_{\max }$ will be sometimes omitted in order to ease the notation.

\section{Proofs of Theorem 1.3 and Theorem 1.5}

\section{Proof of Theorem 1.3}

For the proof we follow the steps given by McDuff and Tolman in [16] where they compute the absolute Seidel element of $S^{1}$-Hamiltonian actions. Let $\sigma_{x}^{\text {rel }}$ denote the relative section class associated to a fixed point $x \in L$. The lemma below follows from Lemma 2.1 and Lemma 3.2.

Lemma 4.1 Let $\gamma \in \mathcal{P}$ with Hamiltonian $H$. If $x \in L$ is a fixed point of the Hamiltonian circle action of $\gamma$, then

$$
\mu_{v}\left(\sigma_{x}^{\mathrm{rel}}\right)=w(x) \quad \text { and } \quad \tau_{\gamma}\left(\sigma_{x}^{\mathrm{rel}}\right)=-H(x) .
$$


Proof Let $\sigma_{x}^{2}$ denote the class section in $P_{\gamma^{2}}$ corresponding to $\sigma_{x}^{\text {rel }}$. Recall that, by definition, $\gamma^{2}$ has Hamiltonian $2 H$. Thus, by Lemma 2.1 we have

$$
c_{v}\left(\sigma_{x}^{2}\right)=w(x) \text { and } \tau_{\gamma^{2}}\left(\sigma_{x}^{2}\right)=-2 H(x) .
$$

By Lemma 3.2,

$$
c_{v}\left(\sigma_{x}^{2}\right)=\mu_{v}\left(\sigma_{x}^{\mathrm{rel}}\right) \quad \text { and } \quad \tau_{\gamma^{2}}\left(\sigma_{x}^{2}\right)=2 \tau_{\gamma}\left(\sigma_{x}^{\mathrm{rel}}\right),
$$

and the two equalities follow.

Let $\gamma \in \mathcal{P}, \tau \in \mathcal{T}(\gamma)$, and construct $J_{P} \in \mathcal{J}\left(P, \omega, \tau, \Omega_{c}\right)$ from an $S^{1}$-invariant $J \in \mathcal{J}(M, \omega)$ via the doubling procedure, that is, $J_{P}$ is the pullback of an $S^{1}$ invariant almost complex structure on $P_{\gamma^{2}}$ under the embedding $\iota_{2}: P_{\gamma}^{\text {half }} \rightarrow P_{\gamma^{2}}$. Fix $B \in H_{2}^{D}(M, L)$, and consider the moduli space of $J_{P}$-pseudoholomorphic disks with no marked points,

$$
\mathcal{M}\left(P_{\gamma}^{\text {half }}, \sigma_{\max }+B, J_{P}\right) .
$$

Its compactification $\overline{\mathcal{M}}\left(P_{\gamma}^{\text {half }}, \sigma_{\max }+B, J_{P}\right)$ consists of stable maps with only one $J_{P}$-holomorphic section component, which we call the root, and the other components are $J_{P}$-holomorphic disks contained in some fibers of $P_{\gamma}^{\text {half }}$ that we will call bubbles. If the root represents the section class $\sigma^{\prime} \in H_{2}\left(P_{\gamma}^{\text {half }}, N\right)$ and the bubbles represent fiber classes $B_{i} \in H_{2}(M, L), i \in A$, we further have that

$$
\sigma_{\max }+B=\sigma^{\prime}+\sum_{i \in A} B_{i} .
$$

Proposition 4.2 If $B \neq 0$ and $\omega(B) \leq 0$, the moduli space $\overline{\mathcal{M}}\left(P_{\gamma}^{\text {half }}, \sigma_{\max }+B, J_{P}\right)$ is empty. Moreover, if $B=0$, then $J_{P}$ is regular, and the moduli space $\mathcal{M}\left(P_{\gamma}^{\text {half }}, \sigma_{\max }, J_{P}\right)$ is compact and can be identified with $L \cap F_{\max }$.

Proof We begin with the first assertion. It is sufficient to show that for a $J_{P}$-holomorphic section with boundary on $N$ representing a section class $\sigma=\sigma_{\max }+B$ one has

$$
\Omega_{c}(\sigma) \geq \Omega_{c}\left(\sigma_{\max }\right),
$$

with equality only if $B=0$. Indeed, suppose (4-1) holds and assume there is a $J_{P}$-holomorphic section representing $\sigma_{\max }+B$, with $B \neq 0$ and $\omega(B) \leq 0$. Then

$$
\Omega_{c}(\sigma)=\Omega_{c}\left(\sigma_{\max }+B\right)=\Omega_{c}\left(\sigma_{\max }\right)+\omega(B) \leq \Omega_{c}\left(\sigma_{\max }\right) .
$$

This is impossible by (4-1) unless $\omega(B)=0$. This latter condition implies that $B=0$, which contradicts $B \neq 0$. Let us now prove (4-1). 
Fix a point $[z, p] \in P_{\gamma}^{\text {half }}$ and consider $w \in T_{[z, p]} P_{\gamma}^{\text {half }}$. Write $w=h+v$, where $h$ and $v$ are respectively the horizontal and vertical parts of $w$. Choose $c>0$ such that $c>H_{\max }$. Then

$$
\begin{aligned}
& \Omega_{c}\left(w, J_{P} w\right) \\
& \quad=\left(\omega-d H \wedge d x-d H \wedge d y-\frac{H}{\pi} d x \wedge d y+\frac{c}{\pi} d x \wedge d y\right)\left(v+h, J v+J_{0} h\right) \\
& \quad=\omega_{p}(v, J v)+\frac{(c-H(p))}{\pi} d x \wedge d y\left(h, J_{0} h\right) \\
& \quad \geq \frac{c-H_{\max }}{\pi} d x \wedge d y\left(h, J_{0} h\right) .
\end{aligned}
$$

where the last inequality holds since $J$ is $\omega$-tame. Since $\frac{1}{\pi} d x \wedge d y$ evaluates to 1 on the disk of radius 1 ,

$$
\Omega_{c}(\sigma) \geq\left(c-H_{\max }\right)=\Omega_{c}\left(\sigma_{\max }\right)
$$

for a $J_{P}$-holomorphic section with boundary on $N$ representing a section class $\sigma=\sigma_{x}^{\text {rel }}+B$, with $x$ some fixed point. Note that equality in (4-2) only occurs when the vertical part of $w$ vanishes. Hence, equality holds only when $B=0$ and $x \in F_{\max } \cap L$.

Next, we show that $\mathcal{M}\left(P_{\gamma}^{\text {half }}, \sigma_{\max }, J_{P}\right)$ is compact (ie that it coincides with its compactification $\left.\overline{\mathcal{M}}\left(P_{\gamma}^{\text {half }}, \sigma_{\max }, J_{P}\right)\right)$ and that it can be identified with $L \cap F_{\max }$. Consider a stable map representing $\sigma$. Such a stable map consists of exactly one root $\sigma^{\prime}$ and possibly many bubbles representing classes $B_{i}, i \in A$, with positive $\omega$-area. It follows that the only stable maps representing a class $\sigma$ such that

$$
\Omega_{c}(\sigma) \leq\left(c-H_{\max }\right)
$$

are the constant sections $\sigma_{x}^{\text {rel }}$, with $x \in F_{\max } \cap L$, which proves the claim.

It remains to show that $J_{P}$ is regular for $\sigma_{\max }$. Let $\mathcal{B}$ denote the set of smooth maps $u:\left(D^{2}, \partial D^{2}\right) \rightarrow\left(P_{\gamma}^{\text {half }}, N\right)$ representing the class $\sigma_{\max }$. For $u \in \mathcal{B}$, set

$$
\mathcal{E}_{u}:=C^{\infty}\left(\Lambda_{J_{P}}^{0,1}\left(D^{2}, u^{*} T P_{\gamma}^{\text {half }}\right)\right) \text { and } \mathcal{E}:=\bigsqcup_{u \in \mathcal{B}} \mathcal{E}_{u} .
$$

We have to show that the linearization of

$$
\bar{\partial}_{J_{P}}: \mathcal{B} \rightarrow \mathcal{E}, \quad u \mapsto d u+J_{P} \circ d u \circ j
$$

is surjective at every $u \in \overline{\mathcal{M}}\left(P_{\gamma}^{\text {half }}, \sigma_{\max }, J_{P}\right)$ (at least between suitable completions of the source and the target). Up to completion, this linearization is given by

$$
L_{\bar{\partial}_{J_{P}}, u}: C^{\infty}\left(u^{*} T P_{\gamma}^{\text {half }}, u^{*} T N\right) \rightarrow C^{\infty}\left(\Lambda^{0,1}\left(D^{2}, u^{*} T P_{\gamma}^{\text {half }}\right)\right)
$$


Since we only consider sections one only needs to verify that

$$
L \frac{v}{\partial}_{J_{P}, u}: C^{\infty}\left(u^{*} \operatorname{Vert}, u^{*}(T N \cap \operatorname{Vert})\right) \rightarrow C^{\infty}\left(\Lambda^{0,1}\left(D^{2}, u^{*} \text { Vert }\right)\right) .
$$

We show that the partial indices of the holomorphic bundle pair $\left(u^{*} T P_{\gamma}^{\text {half }}, u^{*} T N\right)$ must be greater than or equal to -1 . Then we conclude by applying the results of Y-G Oh in [17]. Let $u$ be a $J_{P}$-holomorphic section representing $\sigma_{x}^{\text {rel }}$ with $x \in F_{\max }$. Since $x$ is a fixed point, $u^{*}$ Vert reduces to $T_{x} M \cong \mathbb{C}^{n}, u^{*}(T N \cap$ Vert) reduces to $T_{x} L \cong \mathbb{R}^{n}$ and the restriction of $u$ to $S^{1}$ defines a loop of lagrangian subspaces in $\mathbb{C}^{n}$. Moreover, this loop is given by

$$
\left.T L\right|_{u\left(e^{i 2 \pi t}\right)} \equiv d \gamma(t) T_{x} L \subset T_{x} M .
$$

Since the action is semifree, and since $J_{P}$ comes from an $S^{1}$-invariant almost complex structure $J$ on $(M, \omega), d \gamma(t): \mathbb{C}^{n} \rightarrow \mathbb{C}^{n}$ takes the following diagonal expression after an appropriate change of basis of $\mathbb{C}^{n}$ :

$$
d \gamma(t):=\left(\begin{array}{cccc}
e^{i \pi m_{1} t} & 0 & \cdots & 0 \\
0 & \ddots & \ddots & \vdots \\
\vdots & \ddots & \ddots & 0 \\
0 & \cdots & 0 & e^{i \pi m_{n} t}
\end{array}\right),
$$

where $m_{1}, \ldots, m_{n}$ are the weights of the action at $x$ and are given by $m_{1}=\cdots=m_{l}=0$, $l=\operatorname{dim} F_{\max }^{L}$, and $m_{l+1}=\cdots=m_{n}=-1$. By projecting on each factor of $\mathbb{C}^{n}$, the initial Riemann-Hilbert problem splits into a direct sum of 1-dimensional RiemannHilbert problems of the form

$$
\left\{\begin{array}{l}
\bar{\partial} \xi_{j}(z)=0 \text { on } D^{2}, \\
\xi_{j}\left(e^{2 \pi i t}\right) \in \mathbb{R}\left\langle e^{i \pi m_{j} t} \partial / \partial x_{j}\right\rangle,
\end{array}\right.
$$

where $\xi_{j}$ denotes the projection of $\xi: D^{2} \rightarrow \mathbb{C}^{n}$ to the $j^{\text {th }}$ factor, and where the $x_{j}$ are the real coordinates in $\mathbb{C}^{n}$. In this situation the partial indices coincide with the weights (Maslov indices) of each summand. Here the partial indices are all greater than -1 , and Oh [17] proved that regularity holds for holomorphic disks with partial indices greater than -1 , which completes the proof.

Lemma 4.1 gives the general form of the terms in the Seidel element. Proposition 4.2 then ends the proof of the first part of Theorem 1.3 since it gives the specific form of the leading term of the Seidel element, and since it shows that the sum runs over the fiber classes $B$ with $\omega(B)>0$. To end the proof of Theorem 1.3, we show the vanishing of all the other terms provided $F_{\max }$ is of codimension 2. 
Proposition 4.3 If $\operatorname{codim}_{M} F_{\max }=2$, then $a_{B}=0$ for all $B \in \pi_{2}(M, L)$ with $\mu_{L}(B)>0$.

Proof This is done by a simple dimension argument. Note that for $\sigma=\sigma_{\max }+B$, the moduli space $\mathcal{M}^{\text {pearl }}\left(\left[L_{-}\right], x_{+}, \sigma\right)$ is empty unless

$$
\left|x_{+}\right|=n+\mu_{v}\left(\sigma_{\max }\right)+\mu_{L}(B) .
$$

From Lemma 4.1 and (4-3) we have

$$
n=\operatorname{dim} F_{\max }^{L}-w_{\max }=\operatorname{dim} F_{\max }^{L}-\mu_{v}\left(\sigma_{\max }\right),
$$

implying that $\left|x_{+}\right|=\operatorname{dim} F_{\max }^{L}+\mu_{L}(B)$. Now, taking $B$ with $\mu_{L}(B)>0$, one must have $\mu_{L}(B) \geq 2$ by monotonicity, so that $\left|x_{+}\right|>n$ if $\operatorname{codim}_{M} F_{\max }=2$ or equivalently $\operatorname{codim}_{L} F_{\text {max }}^{L}=1$.

\section{Proof of Theorem 1.5}

The idea here is to adapt Akveld and Salamon's line of proof for length-minimizing exact lagrangian loops in $\mathbb{C} P^{n}$ (see [1]). We will make use of the following general result they showed:

Proposition 4.4 [1, Lemmas 5.3 and 5.4] Let $\gamma \in \mathcal{P}_{L} \operatorname{Ham}(M, \omega)$ with $\gamma \in \mathcal{P}$. Let $A \in \pi_{2}\left(P_{\gamma}^{\text {half }}, N\right)$ be a section class. Suppose that for any $\tau \in \mathcal{T}^{ \pm}(\gamma)$ there exists a family $J=\left\{J_{z}\right\}_{z \in D^{2}}$ of $\omega$-tame almost complex structures in $M$ such that the moduli space $\mathcal{M}\left(P_{\gamma}^{\text {half }}, A ; \tau, \pm J\right)$ is not empty. Then

$$
\epsilon^{+}\left(\tau_{0}, N\right) \geq-\left\langle\left[\tau_{0}\right], A\right\rangle \quad \text { and } \quad \epsilon^{-}\left(\tau_{0}, N\right) \leq-\left\langle\left[\tau_{0}\right], A\right\rangle
$$

for any connection 2 -form $\tau_{0} \in \mathcal{T}(\gamma)$.

We begin by observing that in Proposition 4.2 the results are independent of the choice of connection 2-form in $\mathcal{T}(\gamma)$. Note that the arguments in this proposition apply to show that $J_{P}$ is regular for $\sigma_{\min }$, assuming the minimum fixed point set $F_{\min }$ to be semifree. Also, $\overline{\mathcal{M}}\left(P_{\gamma}^{\text {half }}, \sigma_{\min }, J_{P}\right)$ is nonempty and coincides with $F_{\min } \cap L$.

With this in mind, one argues as follows. Assume $\tau \in \mathcal{T}^{+}(\gamma)$. Then there is a regular $J_{P}^{+}$ such that $\overline{\mathcal{M}}\left(P_{\gamma}^{\text {half }}, \sigma_{\max }, J_{P}^{+}\right)$is nonempty. Similarly, assuming $\tau \in \mathcal{T}^{-}(\gamma)$, there is a regular $J_{P}^{-}$such that $\overline{\mathcal{M}}\left(P_{\gamma}^{\text {half }}, \sigma_{\min }, J_{P}^{-}\right)$is nonempty. Let $\tau_{0}=\tau_{\gamma}$. Then by 
Proposition 4.4 one has

$$
\begin{aligned}
\epsilon(N) & =\epsilon^{+}\left(\tau_{\gamma}, N\right)-\epsilon^{-}\left(\tau_{\gamma}, N\right) \\
& \geq-\left\langle\left[\tau_{\gamma}\right], \sigma_{\max }\right\rangle+\left\langle\left[\tau_{\gamma}\right], \sigma_{\min }\right\rangle \\
& \geq\left\langle\left[\omega-\frac{H}{\pi} d x \wedge d y-d H \wedge d x-d H \wedge d y\right], \sigma_{\min }-\sigma_{\max }\right\rangle \\
& \geq-\left(H_{\min }-H_{\max }\right) \\
& =\ell(N)
\end{aligned}
$$

since the extrema of $H$ are reached on $N$. It follows from [1, Theorem B] that

$$
\ell(N) \leq \epsilon(N) \leq v(N),
$$

hence the proof.

\section{Application to monotone toric manifolds}

\section{Toric manifolds: the Delzant construction}

The following is taken from [20], [6] or [9]. Let $\langle\cdot, \cdot\rangle: \mathbb{R}^{n} \times\left(\mathbb{R}^{n}\right)^{*} \rightarrow \mathbb{R}$ denote the standard pairing. Symplectic toric manifolds are compact connected symplectic manifolds $\left(M^{2 n}, \omega\right)$ together with an effective Hamiltonian action of $\mathbb{T}^{n}$ and a choice of corresponding moment map $\mu$. It is well-known that the image $\Delta:=\mu(M) \subset\left(\mathbb{R}^{n}\right)^{*}$ is a convex polytope, meaning that this is an intersection of a collection of affine halfplanes in $\left(\mathbb{R}^{n}\right)^{*}$. Such half-planes are determined by vectors $\left\{v_{i}\right\}_{i \in 1, \ldots, d}$ in $\mathbb{R}^{n}$ and real numbers $\left\{a_{i}\right\}_{i \in 1, \ldots, d}$. Explicitly, the polytope is given by

$$
\Delta:=\left\{f \in\left(\mathbb{R}^{n}\right)^{*} \mid\left\langle f, v_{i}\right\rangle \geq a_{i}, 1 \leq i \leq d\right\} .
$$

The $v_{i}$ represent inward-pointing normal vectors to the facets, ie the codimension-1 faces, of the polytope, and the faces of $\Delta$ are in bijection with the sets

$$
F_{I}:=\left\{f \in\left(\mathbb{R}^{n}\right)^{*} \mid\left\langle f, v_{i}\right\rangle=a_{i}, i \in I\right\}, \quad I \subset\{1, \ldots, n\}, \quad F_{I} \neq \varnothing .
$$

Symplectic toric manifolds are in one-to-one correspondence with Delzant polytopes, ie polytopes satisfying the following conditions:

(1) Each vertex has $n$ edges.

(2) The edges at any vertex $p$ are rational in the sense that they are given by some $p+t f_{i}$ with $t \in[0,1]$ and $f_{i} \in \mathbb{Z}^{n}, i=1, \ldots, n$.

(3) At each vertex the corresponding vectors $f_{1}, \ldots, f_{n}$ can be chosen to be a $\mathbb{Z}$-basis of $\mathbb{Z}^{n}$. 
The symplectic toric manifold $M$ with moment polytope $\Delta$ can be realized as a symplectic reduction of a Hamiltonian torus action of $\mathbb{T}^{d-n}$ on $\left(\mathbb{C}^{d}, \omega_{\mathrm{st}}\right)$. The construction is as follows. Let $\left\{e_{i}\right\}_{i=1, \ldots, d}$ denote the standard basis of $\mathbb{R}^{d}$. It is easy to see that the map $\pi: \mathbb{R}^{d} \rightarrow \mathbb{R}^{n}, e_{i} \mapsto v_{i}$ descends to a surjective Lie group morphism

$$
\pi: \mathbb{T}^{d} \rightarrow \mathbb{T}^{n}
$$

Let $N:=\operatorname{ker} \pi$. If $\iota: N \rightarrow \mathbb{T}^{d}$ denotes the inclusion, then the composition of $\iota$ with the standard Hamiltonian action of $\mathbb{T}^{d}$ on $\mathbb{C}^{d}$,

$$
\left(e^{i \theta_{1}}, \ldots, e^{i \theta_{d}}\right) \cdot\left(z_{1}, \ldots, z_{d}\right)=\left(e^{-2 \pi i \theta_{1}} z_{1}, \ldots, e^{-2 \pi i \theta_{d}} z_{d}\right),
$$

gives a Hamiltonian action of $\mathbb{T}^{d-n}$ on $\mathbb{C}^{d}$. Let $\left\{w_{1}, \ldots, w_{d-n}\right\} \subseteq \operatorname{ker} \pi$ be a basis, where $w_{i}=\sum_{j=1}^{d} w_{i}^{j} e_{j}$. Then

$$
\exp \left(w_{i}\right) \cdot\left(z_{1}, \ldots, z_{d}\right)=\left(e^{-2 \pi i w_{i}^{1}} z_{1}, \ldots, e^{-2 \pi i w_{i}^{d}} z_{d}\right) .
$$

Furthermore, considering the exact sequence of dualized Lie algebras

$$
0 \rightarrow\left(\mathbb{R}^{n}\right)^{*} \stackrel{\pi^{*}}{\rightarrow}\left(\mathbb{R}^{d}\right)^{*} \stackrel{\iota^{*}}{\rightarrow}(\operatorname{Lie}(\operatorname{ker} \pi))^{*} \rightarrow 0
$$

and setting

$$
\rho_{j}:=\iota^{*} e_{j}^{*}, \quad j=1, \ldots, d
$$

the action becomes

$$
\exp (w) \cdot\left(z_{1}, \ldots, z_{d}\right)=\left(e^{-2 \pi i\left\langle\rho_{1}, w\right\rangle} z_{1}, \ldots, e^{-2 \pi i\left\langle\rho_{d}, w\right\rangle} z_{d}\right) .
$$

The moment map of this action is then given by the composition $\iota^{*} \circ \mu_{\mathrm{st}}$, where

$$
\mu_{\mathrm{st}}\left(z_{1}, \ldots, z_{d}\right)=\left(\pi\left|z_{1}\right|^{2}, \ldots, \pi\left|z_{d}\right|^{2}\right)+\left(a_{1}, \ldots, a_{d}\right) .
$$

Explicitly, one gets

$$
\begin{aligned}
\iota^{*} \circ \mu_{\mathrm{st}}\left(z_{1}, \ldots, z_{d}\right) & =\iota^{*}\left(\sum_{i=1}^{d}\left(\pi\left|z_{i}\right|^{2}+a_{i}\right) e_{i}^{*}\right) \\
& =\sum_{i=1}^{d}\left(\pi\left|z_{i}\right|^{2}+a_{i}\right) \rho_{i} \\
& =\sum_{i=1}^{d} \sum_{m=1}^{d-n}\left(\pi\left|z_{i}\right|^{2}+a_{i}\right) w_{m}^{i} w_{m}^{*} .
\end{aligned}
$$


Then 0 is a regular value for $\iota^{*} \circ \mu_{\text {st }}$. Moreover, ker $\pi$ acts freely on the compact submanifold $Z:=\left(\iota^{*} \circ \mu_{\mathrm{st}}\right)^{-1}(0)$. It follows that

$$
M=\left(\iota^{*} \circ \mu_{\mathrm{st}}\right)^{-1}(0) / N
$$

is a compact manifold. Let $\iota_{Z}: Z \rightarrow \mathbb{C}^{d}$ denote the inclusion map and $p_{M}: Z \rightarrow M$ denote the quotient map. Then, by the Marsden-Weinstein theorem, $M$ is equipped with a canonical symplectic structure $\omega$ such that

$$
p_{M}^{*} \omega=\iota_{Z}^{*} \omega_{0} .
$$

With respect to $\omega$, the action of the $n$-torus $\mathbb{T}^{n}=\mathbb{T}^{d} / N$, which leaves $Z$ invariant, is Hamiltonian. The corresponding moment map $\mu$ is defined by

$$
\mu_{\mathrm{st}} \circ \iota_{Z}=\pi^{*} \circ\left(\mu \circ p_{M}\right)
$$

and has image $\Delta$.

\section{Alternative construction of the toric manifold}

Here we describe an alternative construction of the toric manifold $M$ as a complex manifold.

Extend the previously seen map $\pi: \mathbb{R}^{d} \rightarrow \mathbb{R}^{n}$ to a mapping

$$
\pi_{\mathbb{C}}: \mathbb{C}^{d} \rightarrow \mathbb{C}^{n} .
$$

Note that $\pi_{\mathbb{C}}$ sends the standard lattice $\mathbb{Z}^{d}$ to the set of primitive integral generators of the facets of $\Delta$. Hence it induces a map between complex tori $\pi_{\mathbb{C}}: \mathbb{T}_{\mathbb{C}}^{d} \rightarrow \mathbb{T}_{\mathbb{C}}^{n}$. Let $N_{\mathbb{C}}$ denote the kernel of $\pi_{\mathbb{C}}$ so that we have an exact sequence of complex groups

$$
0 \rightarrow N_{\mathbb{C}} \rightarrow \mathbb{T}_{\mathbb{C}}^{d} \rightarrow \mathbb{T}_{\mathbb{C}}^{n} \rightarrow 0 .
$$

Consider now the linear diagonal action $\kappa$ of $\mathbb{T}_{\mathbb{C}}^{d}$ on $\mathbb{C}^{d}$ given by

$$
\kappa(\exp w) \cdot\left(z_{1}, \ldots, z_{d}\right)=\left(\exp \left(w_{1}\right) z_{1}, \ldots, \exp \left(w_{d}\right) z_{d}\right) .
$$

For any subset $I=\left\{i_{1}, \ldots, i_{k}\right\} \subset\{1, \ldots, d\}$ set

$$
\mathbb{C}_{I}^{d}:=\left\{z \in \mathbb{C}^{d} \mid z_{i}=0 \Longleftrightarrow i \in I\right\} .
$$

Note that this set is a $\mathbb{T}_{\mathbb{C}}^{d}$-orbit and every $\mathbb{T}_{\mathbb{C}}^{d}$-orbit is actually of this type. Now consider the following subspace of $\mathbb{C}^{d}$ :

$$
\mathbb{C}_{\Delta}^{d}:=\bigcup_{\left\{I \mid F_{I} \text { is a face of } \Delta\right\}} \mathbb{C}_{I}^{d} .
$$


This open subset of $\mathbb{C}^{d}$ is in fact the biggest subset on which $N_{\mathbb{C}}$ has no singular orbit: in fact $N_{\mathbb{C}}$ acts on $\mathbb{C}_{\Delta}^{d}$ freely and properly [9].

The corresponding quotient manifold $\mathbb{C}_{\Delta}^{d} / N_{\mathbb{C}}$ is compact and complex. Moreover, the $\mathbb{T}_{\mathbb{C}}^{d}$-action on $\mathbb{C}_{\Delta}^{d}$ induces a $\mathbb{T}_{\mathbb{C}}^{n}$-action on the quotient. This is the quotient that corresponds to $M$. The relation between the two constructions is expressed in the following theorem:

Theorem 5.1 The manifold $Z$ is contained in $\mathbb{C}_{\Delta}^{d}$ and the restriction of an $N_{\mathbb{C}}$-orbit to $Z$ is an $N$-orbit.

\section{The antisymplectic involution}

This involution is the one induced by complex conjugation in $\mathbb{C}^{d}$. In fact, complex conjugation is well-defined on the subset $\mathbb{C}_{\Delta}^{d}$. Furthermore, it commutes with the action of $N_{\mathbb{C}}$ since for every $w \in \mathbb{T}_{\mathbb{C}}^{d}$ we have

$$
\overline{\kappa(w)(z)}=\kappa(\bar{w}) \bar{z} .
$$

Thus it defines an involution on the quotient space

$$
\tau: M \rightarrow M
$$

satisfying

$$
\tau(w \cdot x)=w^{-1} \cdot \tau(x) \text { for all } x \in M, w \in \mathbb{T}^{d} .
$$

That $\tau$ is antisymplectic and that it preserves the moment map of the $\mathbb{T}^{n}$-action on $M$ then follow from the fact that complex conjugation is antisymplectic with respect to the standard symplectic structure and that the moment map associated to the diagonal $\mathbb{T}^{d}$-action on $\mathbb{C}^{d}$ is invariant under this conjugation.

\section{The homology of toric manifolds and their real lagrangians}

In this section we describe the $\mathbb{Z}_{2}$-cohomology rings of the toric manifold $M$ and of its real lagrangian $L=\operatorname{Fix}(\tau)$. We will also explain how these rings are isomorphic, the isomorphism being given by a degree- 2 ring homomorphism.

On the homology of the toric manifold The homology of the toric manifold $M$ is generated by its toric divisors, which correspond to the facets of $\Delta$. If $D_{1}, \ldots, D_{d}$ denote those facets then they are geometrically realized as

$$
D_{k}=Z \cap \mathbb{C}_{k}^{d} .
$$


These determine codimension- 2 cycles in $X$. Let $Y_{1}, \ldots, Y_{d} \in H_{2 n-2}\left(M, \mathbb{Z}_{2}\right)$ denote the homology classes of the corresponding toric divisors. Then

$$
H_{*}\left(M ; \mathbb{Z}_{2}\right)=\frac{\mathbb{Z}_{2}\left[Y_{1}, \ldots, Y_{d}\right]}{P(\Delta)+\operatorname{SR}(\Delta)},
$$

where $P(\Delta)$ and $\operatorname{SR}(\Delta)$ denote the following ideals:

$$
\begin{aligned}
P(\Delta) & :=\left\langle\sum_{k}\left\langle\xi, v_{k}\right\rangle Y_{k} \mid \xi \in\left(\mathbb{Z}_{d}\right)^{*}\right\rangle, \\
\operatorname{SR}(\Delta) & \left.:=\left\langle\prod_{i \in I} Y_{i}\right| I \subset\{1, \ldots, d\} \text { with } D_{I}:=D_{i_{1}} \cap \cdots \cap D_{i_{k}}=\varnothing, I \text { primitive }\right\rangle,
\end{aligned}
$$

where $I$ is primitive if $D_{I \backslash\left\{i_{m}\right\}} \neq \varnothing$ for all $i_{m} \in I$. In this setting the Chern class $c_{1}(M)$ of $M$ is given by the Poincaré dual of $Y_{1}+\cdots+Y_{d}$.

We should also mention that there is a natural isomorphism between $H_{2}\left(M, \mathbb{Z}_{2}\right)$ and the set of tuples $A=\left(a_{1}, \ldots, a_{d}\right) \in \mathbb{Z}^{d}$ such that

$$
\sum_{k} a_{k} v_{k}=0
$$

Under this isomorphism the pairing of $A$ with $\operatorname{PD}\left(Y_{i}\right)$ (the Poincare dual of $Y_{i}$ ) simply coincides with the projection to the $i$-factor of $A$ :

$$
\left\langle A, \operatorname{PD}\left(Y_{i}\right)\right\rangle=a_{i} .
$$

The following result due to $\mathrm{V}$ Batyrev will be useful:

Theorem 5.2 [3] For any primitive $I \in\{1, \ldots, d\}$ there is a unique vector $a_{I}=$ $\left(a_{1}, \ldots, a_{d}\right) \in H_{2}\left(M, \mathbb{Z}_{2}\right)$ such that

$$
a_{k}=1 \text { for all } k \in I, \quad a_{k} \leq 0 \text { for } k \notin I .
$$

The homology of the real lagrangian Let $g$ be a $\tau$-invariant Riemannian metric on $M$. Let $g$ also denote the restriction of $g$ to $L$. Note that for generic $\xi \in \operatorname{Lie}\left(\mathbb{T}^{n}\right)$ the function

$$
f_{\xi}: M \rightarrow \mathbb{R}, \quad x \mapsto\langle\mu(x), \xi\rangle
$$

is Morse. Moreover, there exists a second-category Baire subset of $\tau$-invariant metrics such that the pair $\left(f_{\xi}, g\right)$ is Morse-Smale. Then Crit $f_{\xi}$ corresponds to the vertices of the Delzant polytope, ie the critical points of the moment map. Moreover, for any vertex $p$ the Morse index is given by

$$
|p|_{M}=2 \times \#\{1-\text { dimensional faces } \psi \text { at } p \text { such that }\langle\psi, \xi\rangle<0\},
$$


hence $f_{\xi}$ is perfect. Let $\left.f_{\xi}\right|_{L}$ be the restriction of $f_{\xi}$ to $L$. It is not hard to see that

$$
\text { Crit }\left.f_{\xi}\right|_{L}=\text { Crit } f_{\xi} \text {. }
$$

Furthermore, the restricted pair $\left(\left.f_{\xi}\right|_{L}, g\right)$ is also Morse-Smale. In fact, the inclusion of $L$ into $M$ induces an isomorphism of Morse chain complexes:

Theorem 5.3 (H Duistermaat [7], L Haug [10]) The map

$$
\text { incl: }\left.\mathrm{Crit}_{k} f_{\xi}\right|_{L} \rightarrow \mathrm{Crit}_{2 k} f_{\xi}, \quad p \mapsto p
$$

defines a ring isomorphism between Morse homologies with $\mathbb{Z}_{2}$-coefficients that doubles the degrees:

$$
\text { incl: } H_{*}\left(L, \mathbb{Z}_{2}\right) \stackrel{\cong}{\longrightarrow} H_{2 *}\left(M, \mathbb{Z}_{2}\right) \text {. }
$$

The restriction to $\mathbb{Z}_{2}$-coefficients is essential; it is needed to show that $\left.f_{\xi}\right|_{L}$ is perfect (see [10]). Note that it is easy to find examples where the theorem does not hold for other coefficient rings, for example $\mathbb{R} P^{n}$ in $\mathbb{C} P^{n}$.

\section{The quantum homology of the real lagrangian Set}

$$
P_{L}(\Delta):=\operatorname{incl}^{-1} P(\Delta) \text { and } \operatorname{SR}_{L}(\Delta):=\operatorname{incl}^{-1}(\operatorname{SR}(\Delta)) .
$$

By Theorem 5.3 and from the description of $H_{*}\left(M, \mathbb{Z}_{2}\right)$ we can write

$$
H_{*}\left(L ; \mathbb{Z}_{2}\right)=\frac{\mathbb{Z}_{2}\left[X_{1}, \ldots, X_{d}\right]}{\left\langle P_{L}(\Delta)+\operatorname{SR}_{L}(\Delta)\right\rangle},
$$

where $X_{j}$ is a formal variable of homological degree $n-1$ representing the homology class of $D_{j} \cap L$. Let $I=\left(i_{1}, \ldots, i_{d}\right)$ be a multi-index of nonnegative integers. Then set

$$
X^{I}:=X_{1}^{i_{1}} \cdots X_{d}^{i_{d}},
$$

which has homological degree

$$
n-\sum i_{k}
$$

In general, given pure elements $\tilde{X}_{k} \in H_{n-d_{k}}\left(M, \mathbb{Z}_{2}\right)$ and a multi-index $I=\left(i_{1}, \ldots, i_{d}\right)$, the homological degree of $\tilde{X}^{I}$ is given by

$$
n-\sum_{k} i_{k} d_{k}
$$

According to [5], since the homology of $L$ is generated by the classes of homological degree $n-1$, the lagrangian is either wide, ie there is an identification $\mathrm{QH}\left(L ; \Lambda_{L}\right) \cong$ $H_{*}\left(L ; \mathbb{Z}_{2}\right) \otimes \Lambda_{L}$, or narrow, ie $\mathrm{QH}\left(L ; \Lambda_{L}\right)=0$. Haug [10] showed that the Floer 
differential vanishes for the standard complex structure, which happens to be generic, thus proving that real lagrangians are actually wide:

Theorem 5.4 [10, Theorem A] The real lagrangian $L$ is wide, as $d^{Q}$ generically vanishes. Furthermore, the isomorphism $\mathrm{QH}_{*}\left(L ; \Lambda_{L}\right) \cong H_{*}\left(L ; \mathbb{Z}_{2}\right) \otimes \Lambda_{L}$ is canonical.

By the theorem above each of the $X_{i}$ defines an element in $\mathrm{QH}_{*}\left(L ; \Lambda_{L}\right)$ also denoted $X_{i}$. By Theorem 5.2, for any primitive $I \subset\{1, \ldots, d\}$ there is a unique vector $a_{I}=\left(a_{1}, \ldots, a_{d}\right) \in H_{2}\left(M ; \mathbb{Z}_{2}\right)$ such that

$$
a_{k}=1 \text { for all } k \in I, \quad a_{k} \leq 0 \text { for } k \notin I .
$$

From Theorem 5.3 the same relations exist in $H_{*}\left(L ; \mathbb{Z}_{2}\right)$. Write $I=\left(i_{1}, \ldots, i_{l}\right)$ and let $J=\left(j_{1}, \ldots, j_{m}\right)$ denote the complement of $I$ in $\{1, \ldots, d\}$. Set

$$
\begin{aligned}
P_{L}^{Q}(\Delta) & :=P_{L}(\Delta) \\
\mathrm{SR}_{L}^{Q}(\Delta) & \left.:=\left\langle X_{I}-X_{J}^{\left|a_{J}\right|} q^{\zeta\left(a_{J}\right)}\right| I \text { primitive }\right\rangle,
\end{aligned}
$$

where $I=\left(i_{1}, \ldots, i_{l}\right) \subset\{1, \ldots, d\}, J$ and $a_{I}$ are as above, and we use the notation

$$
X_{I}:=X_{i_{1}} \cdots X_{i_{l}}, \quad X_{J}^{\left|a_{J}\right|}:=X_{j_{1}}^{\left|a_{j_{1}}\right|} \cdots X_{j_{m}}^{\left|a_{j_{m}}\right|}, \quad \zeta\left(a_{J}\right):=-l+\sum_{r=1}^{m}\left|a_{j_{r}}\right| .
$$

The remainder of this section is dedicated to proving the following:

\section{Proposition 5.5}

$$
\mathrm{QH}_{*}\left(L ; \Lambda_{L}\right) \cong \frac{\mathbb{Z}_{2}\left[X_{1}, \ldots, X_{d}\right]\left[q^{-1}, q\right]}{P_{L}(\Delta)+\operatorname{SR}_{L}^{Q}(\Delta)}
$$

First, write

$$
H_{*}\left(L ; \mathbb{Z}_{2}\right)=\frac{\mathbb{Z}_{2}\left[X_{1}, \ldots, X_{d}\right]}{\left\langle f_{1}, \ldots, f_{r}\right\rangle},
$$

where $f_{1}, \ldots, f_{k}$ denote the polynomial relations in the $X_{i}$. For $j=1, \ldots, k$, let $f_{j}^{Q}$ to be the polynomials in the $X_{i}$ variables where the standard product is replaced by the quantum product. The following two results are simple adaptations of [19, Lemmata]. We prove them for convenience:

Lemma 5.6 The elements $X_{1}, \ldots, X_{d}$ generate $\mathrm{QH}_{*}\left(L ; \Lambda_{L}\right)$.

Proof Assume for simplicity that $X_{1}, \ldots, X_{d}$ are critical points of a perfect Morse function on $L$. The proof is by decreasing induction on the homological degree of pure elements, starting at degree $\operatorname{deg}=n$. For $\operatorname{deg}=n$, since $N_{L} \geq 2$, the quantum 
differential $\left.d_{Q}\right|_{R\left\langle\mathrm{Crit}_{n} f\right\rangle}$ restricts to $d_{0}$. Consequently, the unique maximum of $f$ defines an element in $\mathrm{QH}_{n}\left(L ; \Lambda_{L}\right)$, namely $[L]$. Since $[L]$ is generated by the $X_{i}$, the degree- $n$ case follows.

Assume that pure elements of (homological) degree at least deg are generated by $X_{1}, \ldots, X_{d}$ : we show that every monomial $X^{I}$ of degree deg -1 is generated by the $X_{k}$. Let $X^{I, Q}$ denote the element obtained by making the lagrangian quantum product of $X_{k}$ with multi-index $I$. By definition of the quantum product we have that

$$
X^{I, Q}=X^{I}+\sum_{j \geq 1,|R| \geq \operatorname{deg}} \lambda_{R, j} X^{R} q^{-j}, \quad \lambda_{R, j} \in \mathbb{Z}_{2} .
$$

It follows from the induction hypothesis that the $X^{R}$ in the equation above can be written as quantum products of the $X_{k}$; hence the conclusion.

Arguing as in the preceding lemma, we obtain that

$$
f_{j}^{Q}\left(X_{1}, \ldots, X_{d}\right)=f_{j}\left(X_{1}, \ldots, X_{d}\right)+g_{j}^{Q}\left(X_{1}, \ldots, X_{d}\right)=g_{j}^{Q}\left(X_{1}, \ldots, X_{d}\right)
$$

since $f_{j}$ is assumed to be a relation in homology. Let $q_{1}, \ldots, q_{d}$ be abstract variables of degree $\operatorname{deg}\left(q_{k}\right):=n-d_{k}$. Here, it is understood that the degree of $q^{I}$ is given by (5-3) for a multi-index $I=\left(i_{1}, \ldots, i_{d}\right)$. Thus, when evaluated at $\left(X_{1}, \ldots, X_{d}\right)$, the polynomials with $\mathbb{Z}_{2}\left[q, q^{-1}\right]$-coefficients in the $q_{1}, \ldots, q_{d}$ variables

$$
f_{j}^{[\omega]}\left(q_{1}, \ldots, q_{d}\right):=f_{j}^{Q}\left(q_{1}, \ldots, q_{d}\right)-g_{j}^{Q}\left(q_{1}, \ldots, q_{d}\right)
$$

define relations in the quantum homology. We have the following:

$$
\text { Lemma 5.7 } \mathrm{QH}_{*}\left(L ; \Lambda_{L}\right)=\mathbb{Z}_{2}\left[q, q^{-1}\right]\left(\left[X_{1}, \ldots, X_{d}\right]\right) /\left\langle f_{1}^{[\omega]}, \ldots, f_{r}^{[\omega]}\right\rangle .
$$

Proof We have seen that the $f_{j}^{[\omega]}$ define relations in quantum homology. Let $\mathcal{I}$ denote the ideal generated by the $f_{j}^{[\omega]}, j=1, \ldots, r$. We show that any polynomial defining a relation in the quantum homology actually belongs to $\mathcal{I}$. As in the preceding lemma, the proof is by decreasing induction on the degree of the polynomials, in the variables $q_{1}, \ldots, q_{d}$, starting at degree $n$. Let $P^{Q}$ denote a relation of degree $n$. Then, $P^{Q}$ is a constant polynomial $1 \otimes \lambda$ for some $\lambda \in \mathbb{Z}_{2}\left[q, q^{-1}\right]$. We show that $\lambda=0$. Evaluating $P^{Q}$ at $X_{1}, \ldots, X_{d}$ gives $\lambda[L]=0$, which can only happen if $\lambda=0$ since $[L] \neq 0$ ( $L$ being wide). It follows that any degree- $n$ relation is a trivial relation and so belongs to $\mathcal{I}$.

Now, let $P^{Q} \neq 0$ be a polynomial defining a relation in quantum homology where all terms have degree at least $k$, and write

$$
P^{Q}=\sum_{i} P_{i} \otimes q^{d_{i}}+r
$$


where the $P_{i}$ are degree- $k$ polynomials in $q_{1}, \ldots, q_{n}$ with $\mathbb{Z}_{2}$-coefficients, the $d_{i}$ are pairwise different, and the degree of $r$ in $q_{1}, \ldots, q_{n}$ is strictly bigger than $k$. Since $P^{Q}\left(X_{1}, \ldots, X_{d}\right)=0$ one has that $P_{i}\left(X_{1}, \ldots, X_{d}\right)=0$ for all $i$. Hence, there are polynomial functions $\varphi_{i}$ such that $P_{i}=\varphi_{i}\left(f_{1}, \ldots, f_{r}\right)$. Replacing the ordinary product by the quantum homology product gives $\varphi_{i}\left(f_{1}^{[\omega]}, \ldots, f_{r}^{[\omega]}\right)=P_{i}+r^{\prime}$, where $\operatorname{deg} r^{\prime}>k$. Consequently,

$$
P^{Q}=\sum_{i} \varphi_{i}\left(f_{1}^{[\omega]}, \ldots, f_{r}^{[\omega]}\right) \otimes q^{d_{i}}+\left(r-\sum_{i} r^{\prime} q^{d_{i}}\right)
$$

where the term in parentheses has degree (in the $q_{i}$ ) strictly bigger than $k$, as both $r$ and $r^{\prime}$ have. By the induction hypothesis this term is also generated by the relations $f_{1}^{[\omega]}, \ldots, f_{r}^{[\omega]}$, which finishes the proof.

Proof of Proposition 5.5 Now, we show how to use the formula for relative Seidel elements in order to compute $\mathrm{QH}_{*}\left(L ; \Lambda_{L}\right)$. For a primitive $I=\left(i_{1}, \ldots, i_{l}\right) \subset\{1, \ldots, d\}$, let $J=\left(j_{1}, \ldots, j_{m}\right)$ denote its complement in $\{1, \ldots, d\}$. Consider the unique vector $a_{I}=\left(a_{1}, \ldots, a_{d}\right) \in H_{1}\left(L ; \mathbb{Z}_{2}\right)$ such that

$$
a_{k}=1 \text { for all } k \in I, \quad a_{k} \leq 0 \text { for } k \notin I, \quad \sum a_{k} v_{k}=0 .
$$

Let $\Lambda_{j}^{1 / 2}$ denote the half-turn map associated to $\Lambda_{j}$, the $S^{1}$-action generated by the normal to the $j$-facet of the Delzant polytope. From (5-1) and since $\Lambda_{j}^{1 / 2}$ is its own inverse, for all $x \in L$ we have

$$
\tau\left(\Lambda_{j}^{1 / 2} \cdot x\right)=\Lambda_{j}^{1 / 2} \cdot x
$$

Hence, $\Lambda_{j}^{1 / 2} \in \mathcal{P}_{L} \operatorname{Ham}(M, \omega)$, where $\Lambda_{j}^{1 / 2}$ also denotes the path from the identity to the corresponding half-turn map and which is induced from $\Lambda_{j}$. Consequently, in terms of Hamiltonian paths preserving $L$, (5-5) means that

$$
\left(\Lambda_{1}^{1 / 2}\right)^{a_{1}} \ldots\left(\Lambda_{d}^{1 / 2}\right)^{a_{d}}=\mathrm{id}_{L} .
$$

Since we are dealing with a torus action, the order of the terms in the left-hand side of (5-6) can be reorganized in such way that we finally get

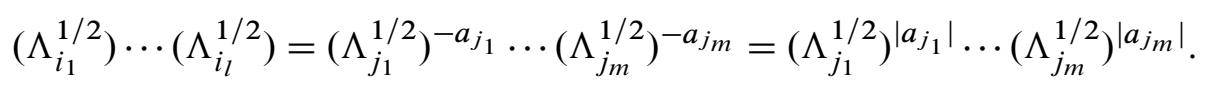

Observe that the maximum fixed point set of each $\Lambda_{j}$ is given by the divisor corresponding to $D_{j}$, hence is of codimension 2, and both assumptions (A1) and (A2) are verified in this context. It follows from Theorem 1.3 that $S_{L}\left(\Lambda_{j}^{1 / 2}\right)=X_{j} \otimes q$. Thus, considering the lagrangian Seidel element associated to both sides in (5-7), we have

$$
X_{i_{1}} \star \cdots \star X_{i_{l}} \otimes q^{l}=X_{j_{1}}^{\left|a_{j_{1}}\right|} \star \cdots \star X_{j_{m}}^{\left|a_{j m}\right|} \otimes q^{\sum_{r=1}^{m}\left|a_{j_{r}}\right|}
$$


where $\star$ stands for the quantum lagrangian product (see [4]). As a consequence of Lemma 5.7 and of the presentation of $H_{*}\left(L ; \mathbb{Z}_{2}\right)$, these are the only multiplicative relations in $\mathrm{QH}_{*}\left(L ; \Lambda_{L}\right)$. Furthermore, the additive relations are the same as in standard homology. The presentation of $\mathrm{QH}_{*}\left(L ; \Lambda_{L}\right)$ follows.

Remark 5.8 - This presentation is the same as that given in [16, Proposition 5.2] for the toric manifold $(M, \omega)$. Let $\Lambda_{M}:=\mathbb{Z}_{2}\left[Q^{-1}, Q\right]$ with $|Q|=2$, and denote by $\mathrm{QH}_{*}\left(M ; \Lambda_{M}\right)$ the corresponding quantum homology. Then, as above,

$$
\mathrm{QH}_{*}\left(M ; \Lambda_{M}\right) \cong \frac{\mathbb{Z}_{2}\left[Y_{1}, \ldots, Y_{n}\right]\left[Q^{-1}, Q\right]}{P(\Delta)+\mathrm{SR}^{Q}(\Delta)},
$$

where $Y_{i}$ represents the class of the divisor $D_{i}, P(\Delta)$ stands for the set of linear relations between the divisors, and $\mathrm{SR}^{Q}(\Delta)$ stands for the set of quantum multiplicative relations between the divisors. It follows directly that there is a ring isomorphism

$$
\psi: \mathrm{QH}_{*}\left(L ; \Lambda_{L}\right) \rightarrow \mathrm{QH}_{2 *}\left(M ; \Lambda_{M}\right)
$$

such that $\psi\left(X_{i}\right)=Y_{i}$ and $\psi(q)=Q$. This ring isomorphism is actually induced by the inclusion incl in Theorem 5.3, as was shown by Haug in [10]. It is worth noticing that, in the notation above, $\psi\left(S_{L}\left(\Lambda_{j}^{1 / 2}\right)\right)=S\left(\Lambda_{j}\right)$, ie relative Seidel elements associated to loops dual to facets are sent to the corresponding absolute Seidel elements under $\psi$.

- Using the $\mathrm{QH}_{*}\left(M ; \Lambda_{M}\right)$-module structure of the quantum homology of $L$, denoted by $\odot$, one also has $[5 ; 11]$

$$
S_{L}\left(\Lambda_{j}^{1 / 2}\right) \star S_{L}\left(\Lambda_{j}^{1 / 2}\right)=S_{L}\left(\left(\Lambda_{j}^{1 / 2}\right)^{2}\right)=S\left(\Lambda_{j}\right) \odot[L] ;
$$

in other words $S_{L}\left(\Lambda_{j}^{1 / 2}\right)$ is in a sense a square root of $S\left(\Lambda_{j}\right)$. As was pointed out to me by François Charette, these relations completely determine the $\mathrm{QH}_{*}\left(M ; \Lambda_{M}\right)$-module structure of $\mathrm{QH}_{*}\left(L ; \Lambda_{L}\right)$. Moreover, it is possible to recover the $H_{*}(M)$-module structure of $H_{*}(L)$. For instance, when $N_{L} \geq 3$ the quantum product of $X_{i}$ with itself does not admit any quantum correction term. Hence

$$
S_{L}\left(\Lambda_{j}^{1 / 2}\right) \star S_{L}\left(\Lambda_{j}^{1 / 2}\right)=\left(X_{i} \otimes q\right) \star\left(X_{i} \otimes q\right)=\left(X_{i} \star X_{i}\right) q^{2}=X_{i}^{2} q^{2},
$$

and since

$$
S\left(\Lambda_{j}\right) \odot[L]=Y_{i} Q \odot[L]=\left(Y_{i} \odot[L]\right) Q=\left(Y_{i} \odot[L]\right) q^{2},
$$

we get $X_{i}^{2}=Y_{i} \odot[L]$.

We end this section with an example illustrating the results established so far. 
Example 5.9 Consider the pair $\left(\mathbb{C} P^{3}, \mathbb{R} P^{3}\right)$, where $\mathbb{C} P^{3}$ is equipped with the FubiniStudy form $\omega_{\mathrm{FS}}$. Let $(M, L)=\left(\widetilde{\mathbb{C P}}^{3}, \widetilde{\mathbb{R P}}^{3}\right)$ denote the monotone lagrangian blow-up of $\left(\mathbb{C} P^{3}, \mathbb{R} P^{3}\right)$ with symplectic form $\widetilde{\omega}$ (see [18] for the definition). Topologically,

$$
\widetilde{\mathbb{C P}}{ }^{3} \cong \mathbb{C} P^{3} \# \overline{\mathbb{C} P^{3}} \text { and } \widetilde{\mathbb{R} P^{3}} \cong \mathbb{R} P^{3} \# \mathbb{R} P^{3} \text {. }
$$

One can also view $\widetilde{\mathbb{C P}}{ }^{3}$ as the projectivization of the rank- 2 complex bundle

$$
\mathcal{O}(-1) \oplus \mathbb{C} \rightarrow \Sigma,
$$

where $\Sigma \cong \mathbb{C} P^{2}$ denotes the exceptional divisor. From this point of view, $\widetilde{\mathbb{R P}}^{3}$ is a nontrivial $S^{1}$-bundle over $\mathbb{R} P^{2}$. The group $H_{2}\left(M ; \mathbb{Z}_{2}\right)$ is generated by the class $F$ of the fiber of this fibration and the class $E$ of the exceptional curve $(E=L-F$, where $L=\left[\mathbb{C} P^{1}\right]$ is the class of a line). A simple computation shows that $H_{2}^{D}\left(M, L ; \mathbb{Z}_{2}\right)$ is generated by half of $E$ and half of $F$. We will use the same notation to refer to them.

Now, the symplectic form for the blow-up of weight $\lambda$ is given by $[\widetilde{\omega}]=\left[\phi^{*} \omega_{\mathrm{FS}}\right]-\pi \lambda^{2} e$, where $\phi: \widetilde{\mathbb{C P}}^{3} \rightarrow \mathbb{C} P^{3}$ is the blowing-down map, and where $e \in H^{2}\left(\widetilde{\mathbb{C P}}^{3}, \mathbb{Z}\right)$ is the Poincaré dual of $\Sigma$. Monotonicity then forces $\lambda$ to be $\sqrt{2} / 2$. The torus $\mathbb{T}^{3}$ acts in a Hamiltonian way on $\mathbb{C} P^{3}$, as follows:

$$
\left(\theta_{1}, \theta_{2}, \theta_{3}\right) \cdot\left[z_{0}: z_{1}: z_{2}: z_{3}\right]=\left[z_{0}: e^{-2 \pi i \theta_{1}} z_{1}: e^{-2 \pi i \theta_{2}} z_{2}: e^{-2 \pi i \theta_{3}} z_{3}\right] .
$$

The moment map of this action is

$$
\mu\left(\left[z_{0}: z_{1}: z_{2}: z_{3}\right]\right)=\left(\frac{\pi\left|z_{1}\right|^{2}}{\sum_{i=0}^{3}\left|z_{i}\right|^{2}}, \frac{\pi\left|z_{2}\right|^{2}}{\sum_{i=0}^{3}\left|z_{i}\right|^{2}}, \frac{\pi\left|z_{3}\right|^{2}}{\sum_{i=0}^{3}\left|z_{i}\right|^{2}}\right)
$$

so that the moment polytope is given by

$$
\Delta=\left\{\left(x_{1}, x_{2}, x_{3}\right) \in \mathbb{R}^{3} \mid 0 \leq x_{i}, x_{1}+x_{2}+x_{3} \leq \pi\right\} .
$$

This action lifts to a Hamiltonian action of $\mathbb{T}^{3}$ on the blow-up with moment map $\tilde{\mu}$. The corresponding moment polytope can be identified with

$$
\widetilde{\Delta}=\left\{\left(x_{1}, x_{2}, x_{3}\right) \in \mathbb{R}^{3} \mid 0 \leq x_{1}, 0 \leq x_{2}, 0 \leq x_{3} \leq \frac{\pi}{2}, x_{1}+x_{2}+x_{3} \leq \pi\right\} .
$$

Moreover, the restriction of $\tilde{\mu}$ to $\widetilde{R P}^{3}$ has also image $\widetilde{\Delta}$. Now, the outward normals to the facets are

$v_{1}=(-1,0,0), \quad v_{2}=(0,-1,0), \quad v_{3}=(0,0,-1), \quad v_{4}=(0,0,1), \quad v_{5}=(1,1,1)$.

We introduce some more notation. Let $\Lambda_{i}, i=1, \ldots, 5$ denote the semifree Hamiltonian circle action fixing the facets defined by the $v_{i}$. Let $\Lambda_{i}^{1 / 2}$ denote the Hamiltonian path corresponding to half of $\Lambda_{i}$ and let $X_{i}$ be formal variables representing the 
intersection of $L$ with the divisors associated to the facets with normals $v_{i}$. To compute the quantum homology of $L$, first note that

$$
\begin{gathered}
P(\tilde{\Delta})=\left\langle X_{1}=X_{2}=X_{5}, X_{3}=X_{4}+X_{5}\right\rangle, \\
\operatorname{SR}(\tilde{\Delta})=\left\langle X_{1} X_{2} X_{5}=0, X_{4}\left(X_{4}+X_{5}\right)=0\right\rangle .
\end{gathered}
$$

Setting $X=X_{1}$ and $Y=X_{4}$ and applying Proposition 5.5 yields

$$
\mathrm{QH}_{*}\left(L ; \Lambda_{L}\right)=\mathbb{Z}_{2}[X, Y]\left[q^{-1}, q\right] /\left\langle X^{3}=Y q^{-2}, Y(X+Y)=[L] \otimes q^{-2}\right\rangle,
$$

which is indeed isomorphic to $\mathrm{QH}_{*}\left(M ; \Lambda_{M}\right)$.

It is not hard to see that the product $Y X$ has no quantum term. Set $Y X=\partial E$. Then $Y \star Y=\partial E+[L] \otimes q^{-2}$. Thus

$$
S_{L}\left(\left(\Lambda_{4}^{1 / 2}\right)^{2}\right)=\left(S_{L}\left(\Lambda_{4}^{1 / 2}\right)\right)^{2}=(Y \star Y) \otimes q^{2}=E \otimes q^{2}+[L],
$$

and we see a lower-order term appearing. Note however that the action of $\left(\Lambda_{4}\right)^{2}$ is not semifree on the maximum subset.

Finally, we wish to show that lower-order terms may appear when the maximum fixed point set is of codimension strictly greater than 2 in $M$. Consider the circle action $\Lambda$ associated to the combination $v_{1}+v_{2}+v_{4}$. The maximum fixed point set is semifree and corresponds to the point mapped to the intersection $D_{1} \cap D_{2} \cap D_{4}$ under $\tilde{\mu}$. Then we have

$$
S_{L}(\Lambda)=S_{L}\left(\Lambda_{1}^{1 / 2}\right) \star S_{L}\left(\Lambda_{2}^{1 / 2}\right) \star S_{L}\left(\Lambda_{4}^{1 / 2}\right)=(X \star X \star Y) \otimes q^{3}
$$

It is not hard to check that $X \star X$ coincides with the intersection product $X \cdot X=\partial F$. In order to compute the lagrangian quantum product $(X \cdot X) \star Y$, observe that

$$
(X \cdot X) \star Y=[\mathrm{pt}]+\alpha Y \otimes q^{-2}+\beta X \otimes q^{-2}, \quad \alpha, \beta \in\{0,1\}
$$

for dimensional reasons and since $F$ and $E$ are the only effective Maslov-2 classes. By a direct computation one has $\alpha=1$, hence the conclusion.

\section{Lagrangian uniruledness}

Recall that, for a non-narrow lagrangian, $H_{n}(L) \otimes \Lambda_{L}$ embeds in $\mathrm{QH}_{*}\left(L ; \Lambda_{L}\right)$ canonically. We set $Q_{-}$to be the complement:

$$
Q_{-}:=H_{*}\left(\bigoplus_{k<n} \operatorname{Crit}_{k} f \otimes \Lambda_{L}, d Q\right)
$$


Lemma 5.10 Let $L \subset M$ be a closed monotone lagrangian with $N_{L} \geq 2$. Assume that $L$ is not narrow. If $L$ is not lagrangian uniruled, all the invertible elements of $\mathrm{QH}_{*}\left(L ; \Lambda_{L}\right)$ can be written as

$$
\lambda[L]+x, \quad \text { where } \lambda \in \Lambda_{L} \backslash\{0\} \text { and } x \in Q_{-} .
$$

Proof Assuming $L$ is not uniruled, we show that $Q_{-}$is an ideal in $\mathrm{QH}_{*}\left(L ; \Lambda_{L}\right)$. If this is not the case, then there would exist $x \in \mathrm{QH}_{*}\left(L ; \Lambda_{L}\right)$ and $y \in Q_{-}$such that $x \star y$ has a term of the type $r[L] q^{\mu(B)}, r \neq 0$. Here,

$$
r=\#_{2}\left\{\mathcal{M}^{\text {prod }}(\bar{x}, \bar{y} ;[L], B)\right\}
$$

is the number (modulo 2) of pearl configurations used in the definition of the lagrangian quantum product between $x$ and $y$. Here $\bar{x}$ and $\bar{y}$ denote linear combinations of critical points representing the classes $x$ and $y$ (for two given Morse functions), and $\mathcal{M}^{\text {prod }}(\bar{x}, \bar{y} ;[L], B)$ stands for the moduli space of generalized pearl trajectories representing the class $B \in H_{2}(M, L)$, starting at $\bar{x}, \bar{y}$, and exiting at the (unique) maximum of a given third Morse function. We refer to [5] for further details on the quantum lagrangian product.

Note that $B \neq 0$, since otherwise $r=0$, unless both $\bar{x}$ and $\bar{y}$ have index $n$. Now, since any generic point $p$ of $L$ can be chosen to represent the fundamental class, this contradicts the fact that $L$ is uniruled. Thus, $Q_{-}$is an ideal. Furthermore, since the unit $[L]$ cannot belong to $Q-$ unless $\mathrm{QH}_{*}\left(L ; \Lambda_{L}\right)$ vanishes, any invertible must have such a presentation.

Now we can prove Theorem 1.4.

Theorem 1.4 Assume $L$ is not narrow. Then the claim follows from Lemma 5.10 and the fact that $[L]$ does not appear in any lagrangian Seidel element associated to $\gamma$ by Theorem 1.3. If $L$ is narrow, then the fundamental class $[L]$ is a $d^{Q}$-boundary. Since $[L]$ is represented by the unique maximum of some generic Morse function on $L$, this implies that there is a pseudoholomorphic disk through the maximum, which completes the proof.

Remark 5.11 It is worth pointing out that the assumption on the codimension of $F_{\max }$ could probably be weakened. In fact, in the absolute case, the same conclusion as in Theorem 1.4 holds for the ambient manifold if we assume that all pseudoholomorphic curves (for an $S^{1}$-invariant almost complex structure on $M$ ) that intersect $F_{\max }$ but are not entirely contained in the maximal fixed point set have first Chern number bigger than $\operatorname{codim} F_{\max }$ (see [16, Theorem 1.9(iii)]). The argument in the absolute case 
relies heavily on a localization principle that enables one to characterize the (stable) maps contributing to the nonvanishing of higher-order terms in the Seidel element of the $S^{1}$-action. More precisely, McDuff and Tolman observed that contributions come from the fixed points of a $\mathbb{T}^{2}$-action on the (compactified) moduli space of pseudoholomorphic sections in the Hamiltonian fibration induced by the $S^{1}$-action on $M$. Those fixed points are usually not regular as stable maps.

The author thinks that a statement analogous to [16, Theorem 1.9(iii)] should also hold in the present framework, and more precisely that nonvanishing terms in the lagrangian Seidel element should arise from fixed point sets of some $\mathbb{Z}_{2}$-action on the compactified moduli space of pearls (induced from a choice of $S^{1}$-invariant almost complex structure on the ambient space). Nevertheless, the tools used in the present paper are insufficient to provide a rigorous proof of this, the main issue being the lack of regularity of symmetric almost complex structures and a localization principle in the relative case.

\section{References}

[1] M Akveld, D Salamon, Loops of Lagrangian submanifolds and pseudoholomorphic discs, Geom. Funct. Anal. 11 (2001) 609-650 MR1866797

[2] S Anjos, R Leclercq, Seidel's morphism of toric 4-manifolds, preprint (2014) arXiv: 1406.7641

[3] V V Batyrev, Quantum cohomology rings of toric manifolds, from: "Journées de Géométrie Algébrique d'Orsay", Astérisque 218, Société Mathématique de France, Paris (1993) 9-34 MR1265307

[4] P Biran, O Cornea, A Lagrangian quantum homology, from: "New perspectives and challenges in symplectic field theory", (M Abreu, F Lalonde, L Polterovich, editors), CRM Proc. Lecture Notes 49, Amer. Math. Soc. (2009) 1-44 MR2555932

[5] P Biran, O Cornea, Rigidity and uniruling for Lagrangian submanifolds, Geom. Topol. 13 (2009) 2881-2989 MR2546618

[6] D A Cox, S Katz, Mirror symmetry and algebraic geometry, Mathematical Surveys and Monographs 68, Amer. Math. Soc. (1999) MR1677117

[7] J J Duistermaat, Convexity and tightness for restrictions of Hamiltonian functions to fixed point sets of an antisymplectic involution, Trans. Amer. Math. Soc. 275 (1983) 417-429 MR678361

[8] V Guillemin, E Lerman, S Sternberg, Symplectic fibrations and multiplicity diagrams, Cambridge Univ. Press (1996) MR1414677

[9] VW Guillemin, S Sternberg, Supersymmetry and equivariant de Rham theory, Springer, Berlin (1999) MR1689252 
[10] L Haug, On the quantum homology of real Lagrangians in Fano toric manifolds, Int. Math. Res. Not. (2013) 3171-3220 MR3085757

[11] S Hu, F Lalonde, A relative Seidel morphism and the Albers map, Trans. Amer. Math. Soc. 362 (2010) 1135-1168 MR2563724

[12] S Hu, F Lalonde, R Leclercq, Homological Lagrangian monodromy, Geom. Topol. 15 (2011) 1617-1650 MR2851073

[13] F Lalonde, D McDuff, L Polterovich, Topological rigidity of Hamiltonian loops and quantum homology, Invent. Math. 135 (1999) 369-385 MR1666763

[14] D McDuff, D Salamon, Introduction to symplectic topology, 2nd edition, Clarendon Press, New York (1998) MR1698616

[15] D McDuff, J Slimowitz, Hofer-Zehnder capacity and length minimizing Hamiltonian paths, Geom. Topol. 5 (2001) 799-830 MR1871405

[16] D McDuff, S Tolman, Topological properties of Hamiltonian circle actions, Int. Math. Res. Pap. (2006) Art. ID 72826 MR2210662

[17] Y-G Oh, Riemann-Hilbert problem and application to the perturbation theory of analytic discs, Kyungpook Math. J. 35 (1995) 39-75 MR1345070

[18] A Rieser, Lagrangian blow-ups, blow-downs, and applications to real packing, J. Symplectic Geom. 12 (2014) 725-789 MR3333028

[19] B Siebert, G Tian, On quantum cohomology rings of Fano manifolds and a formula of Vafa and Intriligator, Asian J. Math. 1 (1997) 679-695 MR1621570

[20] A Cannas da Silva, Symplectic toric manifolds, from: "Symplectic geometry of integrable Hamiltonian systems”, Birkhäuser, Basel (2003) 85-173 MR2000746

Département de Mathématiques, Cégep Saint-Laurent 625 avenue Sainte-Croix, Montreal, QC H4L 3X7, Canada

chyvrier@cegep-st-laurent.qc.ca

Received: 17 September $2013 \quad$ Revised: 15 October 2015 